\title{
Pathogen Infection and MORC Proteins Affect Chromatin Accessibility of Transposable Elements and Expression of Their Proximal Genes in Arabidopsis
}

\author{
Yogendra Bordiya, ${ }^{1}$ Yi Zheng, ${ }^{2}$ Ji-Chul Nam, ${ }^{1}$ April C. Bonnard, ${ }^{1}$ Hyong Woo Choi, ${ }^{2}$ Bum-Kyu Lee, ${ }^{3}$ \\ Jonghwan Kim, ${ }^{3}$ Daniel F. Klessig, ${ }^{2}$ Zhangjun Fei, ${ }^{2,4}$ and Hong-Gu Kang ${ }^{1}$ \\ ${ }^{1}$ Department of Biology, Texas State University, San Marcos, TX, U.S.A.; ${ }^{2}$ Boyce Thompson Institute for Plant Research, Ithaca, \\ NY, U.S.A.; ${ }^{3}$ Department of Molecular Biosciences, The University of Texas at Austin, U.S.A.; and ${ }^{4}$ USDA Robert W. Holley \\ Center for Agriculture and Health, Tower Road, Ithaca, NY, U.S.A.
}

Accepted 26 July 2016.

\begin{abstract}
To assess the role of MORC1 in epigenetics in relation to plant immunity, genome-wide chromatin accessibility was compared between mock- or Pseudomonas syringae pv. tomato-inoculated wild type (WT) Arabidopsis, the morc1/2 double mutant, or both. Most changes in chromatin accessibility, scored by DNase I hypersensitive sites (DHSs), were located in the promoters of genes and transposable elements (TEs). Comparisons between morc1/2 and WT receiving the same treatment revealed differential DHSs (dDHSs) predominantly associated with heterochromatic TEs. By contrast, comparisons between mock- and $P$. syringae pv. tomato-inoculated plants from the same genotype showed dDHSs associated with biotic and abiotic stress-related genes; a smaller but significant population was in TEs. Moreover, many defense genes, including $P R-1, P R-2$, and $P R-5$, were proximal to $P$. syringae pv. tomato-induced, TE-associated dDHSs. A random subset of these defense genes showed moderately delayed or reduced expression or both in $P$. syringae pv. tomato-infected morc 1/2 as compared with WT. MORC1 was physically bound to chromatin in a $P$. syringae pv. tomato infection-responsive manner at sites dispersed throughout the genome. Notably, silencing of TE-associated dDHSs proximal to these infectioninduced, MORC1-interacting sites led to significant suppression of $P$. syringae pv. tomato-induced transcription of adjacent defense genes, including $P R-1$. These results provide evidence that MORC1 is associated with TEs and suggest that a subset of these TEs may help regulate their proximal defense genes.
\end{abstract}

Plants have evolved a variety of defense mechanisms to protect themselves from potentially pathogenic microorganisms. Upon infection, recognition of pathogen-associated molecular patterns (PAMPs) by extracellular surface receptors

Y. Bordiya and Y. Zheng contributed equally to this work.

Raw DNase-Seq and ChIP-Seq reads have been deposited in the National Center for Biotechnology Information sequence read archive (SRA) under accession number SRP055733.

Corresponding authors: H.-G. Kang; E-mail: kang @txstate.edu and Z. Fei; E-mail: zf25@cornell.edu

*The $\boldsymbol{e}$-Xtra logo stands for "electronic extra" and indicates that seven supplementary figures and fifteen supplementary tables are published online.

๑) 2016 The American Phytopathological Society leads to the activation of PAMP-triggered immunity (PTI). While PTI is sufficient to prevent the further colonization of many microbes, evolutionary selection has led to the appearance of some pathogens containing effectors that suppress PTI. Whether such pathogens can effectively infect the host plant is then determined by whether the plant expresses a resistance (R) protein that recognizes one of these effectors (also termed avirulence [Avr] factors) and induces effector-triggered immunity (ETI) (Jones and Dangl 2006). Following the activation of either ETI or PTI, a variety of defense responses are induced, such as the accumulation of salicylic acid (SA) and the increased expression of certain pathogenesis-related $(P R)$ genes (Vlot et al. 2009). In addition, the activation of plant immunity is associated with large-scale changes in gene expression (Moore et al. 2011; Vlot et al. 2009). This transcriptional reprogramming involves the highly coordinated action of myriad transcription factors and their associated proteins that function to recruit or modulate RNA polymerase II (RNAPII) (Moore et al. 2011). Growing evidence suggests that chromatin modification and remodeling, which regulates the accessibility of DNA to the transcriptional machinery, as well as posttranscriptional regulation of defense-associated mRNAs are essential for this phenomenon (Berr et al. 2012; Ma et al. 2011; Staiger et al. 2013).

Epigenetic gene regulation plays a critical role in cell differentiation and reprogramming. It also maintains genome integrity by silencing the expression of transposable elements (TEs) and other repeat sequences (Boyko and Kovalchuk 2011; Zaratiegui et al. 2007). Whether DNA is packaged as transcriptionally active euchromatin or transcriptionally silent heterochromatin is determined by the interplay between repressive epigenetic marks, such as DNA methylation and specific histone modifications, and chromatin remodeling complexes that reposition, evict, or alter the composition of nucleosomes (Boyko and Kovalchuk 2008; Ma et al. 2011). Evidence supporting a role for epigenetic regulation of plant immune responses comes from the combined demonstrations that i) loss of histone-modifying enzymes, including histone deacetylase (Kim et al. 2008; Wang et al. 2010), histone methyltransferases (Alvarez-Venegas et al. 2007; Berr et al. 2011; De-La-Peña et al. 2012; Palma et al. 2010), or histone ubiquitin-ligase (Dhawan et al. 2009), alters defense-gene expression and resistance to bacterial and fungal pathogens, ii) SA treatment leads to altered histone methylation and acetylation in key defense genes, including $P R-1$ (AlvarezVenegas et al. 2007; Mosher et al. 2006), iii) mutations in several members of the SWI/SNF family of ATP-dependent 
chromatin remodeling factors, including DDM1 (deficient in DNA methylation 1), ARP6 (actin-related protein 6), and PIE1 (photoperiod-independent early flowering 1), either alter defense gene expression, influence plant immunity, or both (Cheng et al. 2013; Dowen et al. 2012; Li et al. 2010; MarchDíaz et al. 2008), and iv) mutants lacking components in the RNA-directed DNA methylation (RdDM) pathway that mediates epigenetic DNA methylation exhibit altered expression of numerous pathogen-responsive genes and enhanced resistance to virulent, avirulent, and nonpathogenic strains of $P$. syringae pv. tomato (Dowen et al. 2012; Yu et al. 2013).

TEs have long been considered transcriptionally inactive junk DNA (Lewin 1986; Probst and Almouzni 2011). However, several recent reports indicate that dynamic changes in TEs can occur, particularly in response to stress (Grandbastien et al. 2005; Ito et al. 2011; Tittel-Elmer et al. 2010). For example, the first comprehensive stress-induced DNA methylation map of the Arabidopsis genome indicated that TEs are differentially methylated in response to biotic stress or SA treatment (Dowen et al. 2012). Moreover, demethylation of TEs following treatment with the bacterial PAMP flg22 correlated with the downregulation of components involved in RdDM and the concomitant activation of TE expression (Yu et al. 2013). SA treatment also led to decreased methylation of some TEs and this corresponded not only with their upregulation, but also with increased expression of neighboring genes (Dowen et al. 2012). In addition, a wide range of TEs that are transcribed in response to stress with stress-responsive transcription, including ONSEN and mPing, have been implicated in inducing neighboring genes (Ito et al. 2011; Makarevitch et al. 2015; Naito et al. 2009; Yasuda et al. 2013). Together, these observations suggest that TEs may play a role in triggering the transcriptional activation of proximal defense genes.

Efforts to elucidate the components required for resistance to Turnip crinkle virus (TCV) in Arabidopsis led us to identify the CRT1 (compromised recognition of TCV) gene (Kang et al. 2008). Sequence analysis revealed that $C R T 1$ contains the ATPase and S5 domains characteristic of microrchidia (MORC) proteins (Kang et al. 2010). Additional members of the Arabidopsis CRT1 family have been identified by other groups and variously named MORC, DMS, or CRT/CRH (Brabbs et al. 2013; Lorković et al. 2012; Moissiard et al. 2012). To avoid further confusion, we use the nomenclature proposed in a previous report and refer to CRT1 and its family members as MORC1-7 (Langen et al. 2014). Through genetic and biochemical analyses, we and others demonstrated that MORC1 is required for multiple layers of immunity, including ETI and PTI, following inoculation by a wide range of plant pathogens (Kang et al. 2008, 2010, 2012; Wang et al. 2011). In addition, MORC 1 is one of very few proteins known to date that physically associates with a large number of immune components, including at least $12 \mathrm{R}$ proteins and the PAMP recognition receptor FLS2 (Langen et al. 2014; Kang et al. 2008, 2010, 2012).

The demonstration that a small subpopulation of MORC1 is present in the nucleus and that nuclear MORC1 levels increase following activation of PTI or ETI in Arabidopsis (Kang et al. 2012) raised the possibility that this protein also has a nuclear function during plant immunity. Consistent with this hypothesis, MORC proteins from a wide variety of eukaryotes and prokaryotes have been implicated in either DNA recombination and repair, chromatin modification, or both (Iyer et al. 2008; Perry and Zhao 2003). Furthermore, MORC1 binds nucleic acids and exhibits $\mathrm{Mn}^{2+}$-dependent endonuclease activity (Kang et al. 2012) as well as ATPase activity (Kang et al. 2008). Mouse MORC1, the first MORC protein identified, is required for meiotic nuclear division (Watson et al. 1998); more recently, it was implicated in TE repression in the male germline (Pastor et al. 2014). Suggesting a similar role for Arabidopsis MORC proteins, one or both MORC1 and MORC6 were identified in three independent screens for mutants defective for gene silencing (Lorković et al. 2012; Moissiard et al. 2012). The derepression of silenced reporter genes and TEs in morcl and morc6 mutants suggests that these proteins are required for epigenetic gene silencing (Brabbs et al. 2013; Lorković et al. 2012; Moissiard et al. 2012). However, it is currently unclear whether MORC1 and MORC6 fulfill their functions by modulating RdDM (Brabbs et al. 2013; Lorković et al. 2012) or by influencing chromatin structure (Moissiard et al. 2012).

In this study, we used both DNase-Seq (Hesselberth et al. 2009) and ChIP (chromatin immunoprecipitation)-Seq (Furey 2012) approaches to investigate the potential role MORC proteins play in regulating gene silencing and the mechanism through which this process impacts plant immunity. Analyses of DNase hypersensitive sites (DHSs) in mock- and P. syringae pv. tomato-inoculated wild type (WT) and morc $1 / 2$ plants revealed that an enriched proportion of differential DHSs (dDHSs) are located in TEs. Strikingly, TE-associated dDHSs induced by $P$. syringae pv. tomato infection were identified near many defenserelated genes. This finding, combined with the demonstration that i) MORC1 is a chromatin-interacting protein, ii) $P$. syringae pv. tomato infection increases MORC1 binding to P. syringae pv. tomato-induced dDHSs associated with genes and TEs, and iii) expression of many defense genes is delayed, reduced, or both in morc 1/2 mutant plants, suggests that MORC proteins modulate plant immune responses by binding TEs and thereby influencing both their expression and that of proximal genes following pathogen infection.

\section{RESULTS}

Pathogen infection did not dramatically alter
the percentage of promoter-associated DHSs
in either WT or MORC-deficient Arabidopsis.

To characterize changes in the chromatin landscape in response to pathogen infection, we prepared and sequenced DNaseSeq libraries from three genotypes: i) WT, ii) morc $1-2$ morc $2-1$ (morc 1/2) (Kang et al. 2010), a double knock-out mutant lacking MORC1 and its closest homolog MORC2, and iii) morc1-2 morc2-1 morc6-3 (morc1/2/6) (Moissiard et al. 2014), a triple knock-out mutant in which morc6 was introduced into the morc 1/2 background. Plants from each genotype were subjected to four different treatments: no treatment (naïve) or inoculation with buffer (mock), virulent $P$. syringae pv. tomato, or avirulent P. syringae pv. tomato carrying AvrRpt2. A total of 554 million reads were obtained. After discarding reads aligned to the Arabidopsis chloroplast genome, a total of 224 million reads were uniquely aligned to the Arabidopsis genome, while 2.4 million reads were aligned with the $P$. syringae pv. tomato genome (Supplementary Table S1), suggesting very little contamination with bacterial DNA. The Pearson correlation coefficient between biological replicates for each combination ranged from 0.76 to 0.97 ; only four of the 36 libraries were lower than 0.85 . Given that independently grown batches of plants were used for the biological triplicates, to reduce false signals, these correlation coefficient values suggest high reproducibility of the data sets (Supplementary Table S2).

A total of 29,450 DHSs were identified in the Arabidopsis genome (Fig. 1; Supplementary Table S3). As expected, the distribution of DHSs was biased toward euchromatin over heterochromatin, clearly displaying a strong correlation with the density of genes (Supplementary Fig. S1). Among the DHSs, approximately $65 \%$ were located within $1 \mathrm{~kb}$ upstream of the transcription start sites (TSSs) or $1 \mathrm{~kb}$ downstream of the transcription termination site (TTS) of protein-coding genes, approximately $18 \%$ were associated with the gene body, such 
as exons, introns, $5^{\prime}$ and $3^{\prime}$ untranslated regions (UTRs), approximately $11 \%$ were located in sequences $1 \mathrm{~kb}$ upstream or downstream of TEs, approximately $1 \%$ were detected within TEs, and the remaining approximate $5 \%$ were in intergenic regions (Fig. 1A; Supplementary Table S4). For protein-coding genes the majority of DHSs $(64 \%)$ were detected within $1 \mathrm{~kb}$ upstream of their TSSs. Similarly, 78\% of the DHSs associated with TEs were located in this region. These data suggest that the identified DHSs are heavily enriched in promoter regions, which are normally located within $1 \mathrm{~kb}$ upstream of the TSS. Pairwise comparisons among untreated naive plants and mockor pathogen-inoculated WT, morcl/2, or morcl/2/6 mutants revealed no dramatic alterations in percentage of DHS at different genomic locations (Fig. 1).

\section{dDHSs induced by $P$. syringae pv. tomato infection or loss of MORC family members were enriched in the promoters of TEs.}

To assess chromatin dynamics in response to one or both pathogen infection or MORC1/2/6 deficiency, we performed pair-wise comparisons of the DHSs identified in the different plant backgrounds in the presence or absence of pathogen infection. The dDHSs identified by this process represent genomic sites at which the level of chromatin accessibility differs within the corresponding comparison. Pathogen infection induced significant changes in chromatin accessibility, since comparisons of DHSs between mock- and pathogen-infected plants within the WT, morc 1/2, or morc 1/2/6 backgrounds revealed hundreds of dDHSs (Fig. 2A; Supplementary Table S5). By contrast, pairwise comparisons of DHSs in naïve versus mock or avirulent versus virulent $P$. syringae pv. tomato identified 17 or fewer dDHSs (Fig. 2A; Supplementary Table S6), indicating that mock inoculation does not substantially alter the DHS pattern present in untreated naive plants and that the DHSs induced by virulent and avirulent $P$. syringae pv. tomato are very similar.

To further investigate whether MORC proteins influence chromatin accessibility, we compared the DHSs identified in different genotypes subjected to the same treatment. Modest numbers of dDHSs, ranging from 59 to 118 (Fig. 2B), were observed in WT versus morc1/2 and WT versus morc $1 / 2 / 6$ comparisons in which the plants were responding to the same treatment. By contrast, the DHS comparisons between morc $1 / 2$ and morc $1 / 2 / 6$ identified very few dDHSs, with a maximum of five. This latter finding is in line with i) RNA-Seq analyses of morc $1 / 2$ and morc $1 / 2 / 6$, which revealed very little difference between these lines (Moissiard et al. 2014), and ii) our findings that morcl/2 and morc $1 / 2 / 6$ exhibit comparable levels of enhanced susceptibility to virulent and avirulent $P$. syringae pv. tomato (Supplementary Fig. S2). Given the small number of dDHSs observed in comparisons between i) naive and mock,
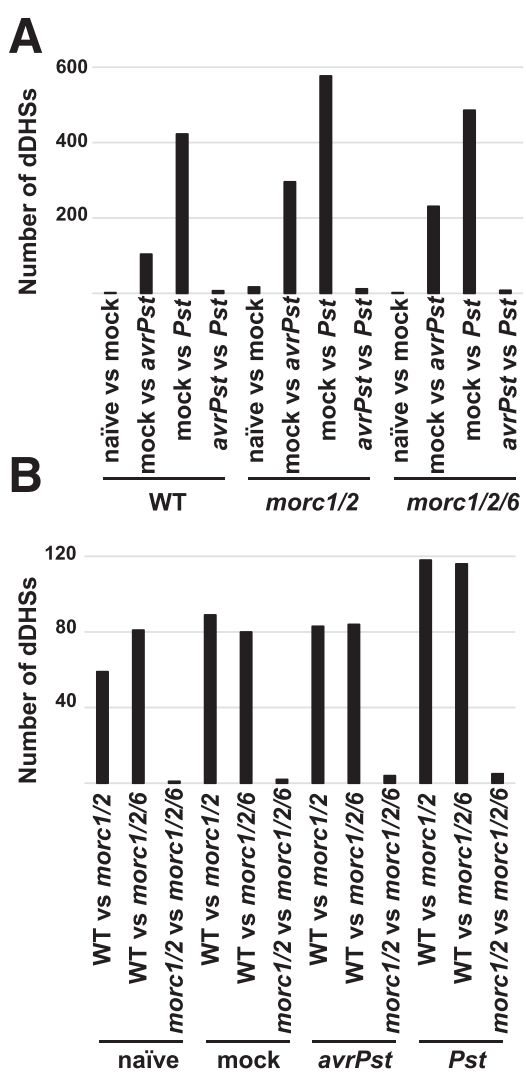

Fig. 2. Pairwise comparisons among naïve, mock-, or Pseudomonas syringae pv. tomato-inoculated wild type (WT) and MORC-deficient plants reveal differential DNase I hypersensitive sites (dDHSs). A, The number of dDHSs detected in the indicated comparisons between different treatments are plotted for each genetic background. B, The number of dDHS from the indicated comparisons between different genetic backgrounds after a particular treatment are plotted.

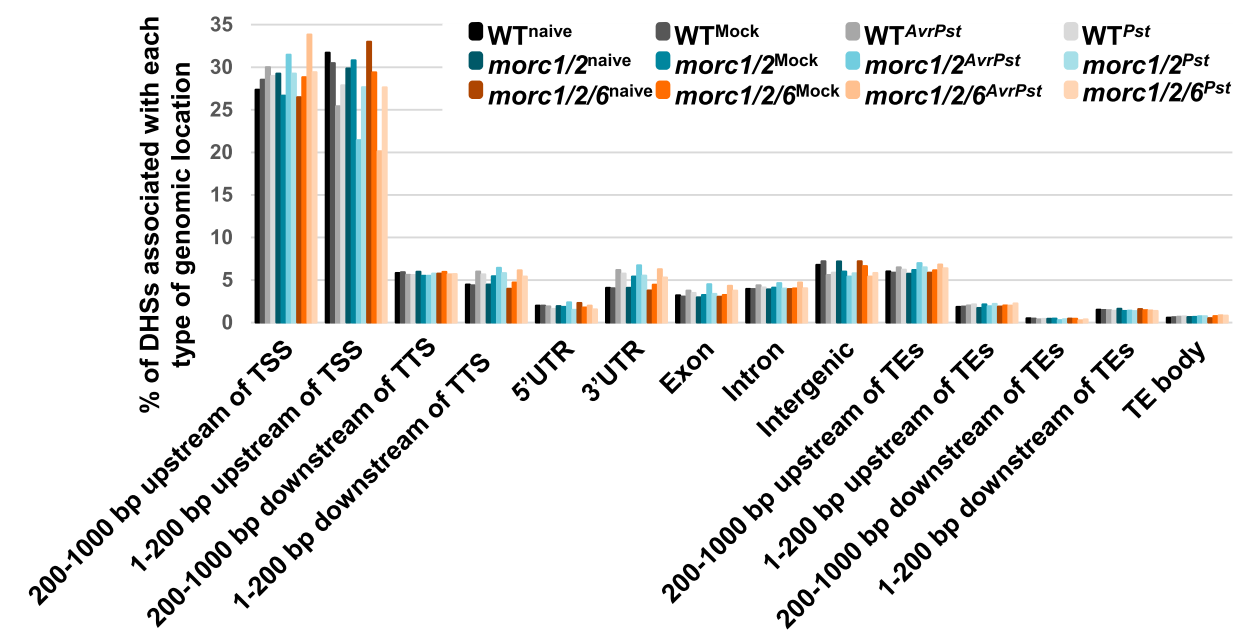

Fig. 1. DNase I hypersensitive sites (DHSs) are predominantly located in the promoter regions of genes and transposable elements (TEs). Genomic locations of DHSs relative to annotated protein-coding genes and TEs. The $y$ axis shows the percentage of DHSs in the indicated locations over the total number of DHSs associated with genes or TEs. TSS, transcription start site; TTS, transcription termination site; UTR, untranslated region. 
ii) avirulent and virulent $P$. syringae pv. tomato, and iii) morc $1 / 2$ and morc $1 / 2 / 6$, only analyses derived from combinations of mock- or P. syringae pv. tomato-inoculated WT plants $\left(\mathrm{WT}^{\text {mock }}\right.$ and $\mathrm{WT}^{P s t}$ ) and mock- or P. syringae pv. tomatoinoculated $\operatorname{morcl} 1 / 2\left(\operatorname{morc} 1 / 2^{\text {mock }}\right.$ and $\left.\operatorname{morc} 1 / 2^{P s t}\right)$ will be presented hereafter (Fig. 2).

Comparisons between the DHSs identified in mock- and pathogen-infected plants within the same genetic background (termed $P$. syringae pv. tomato-induced $\mathrm{dDHSs}$ ) revealed a substantially larger number of dDHSs in genes than in TEs (Fig. 3A). By contrast, DHS comparisons between WT and morcl/2 plants subjected to the same treatment (termed morc 1/2-enhanced dDHSs) identified much lower numbers of dDHSs overall, with slightly more present in TEs than in genes. Interestingly, when the percentage of dDHSs associated with TEs (termed TE-dDHSs) was calculated for each pairwise comparison, a significantly greater number of TE-dDHSs was observed than would be statistically expected (Fig. 3B, indicated as a broken line). Approximately $21 \%$ of the P. syringae pv. tomato-induced dDHSs were associated with TEs in both WT and morcl/2 backgrounds (Fig. 3B). Notably, over $60 \%$ of the morc 1/2-enhanced dDHSs were associated with TEs, irrespective of infection. These striking results suggest that MORC family members participate in modulating the physical accessibility of TE-associated sequences.

To gain insights into the putative biological functions of the gene-associated dDHSs, their gene annotations were analyzed using The Arabidopsis Information Resource (TAIR) database (Fig. 3C; Supplementary Table S7). Of the P. syringae pv. tomato-induced dDHSs, a significant number were associated with 'response to stress' or 'response to biotic/abiotic stress' genes (Fig. 3C, black and dark gray bars). Due to the low number of the morc1/2-enhanced gene dDHSs (43 or fewer), little significance was found in their gene annotations (Fig. 3C).

\section{Members of the MORC family modulated the DNase I accessibility of heterochromatic TEs, while $P$. syringae pv. tomato infection altered the accessibility of TEs distributed throughout the genome.}

TEs are predominantly localized in heterochromatin; these heterochromatic TEs are subject to transcriptional gene silencing primarily via RdDM (Matzke and Mosher 2014). By contrast, the silencing of euchromatic TEs, which also involves DNA methylation, appears to be mediated by a distinct mechanism (Zemach et al. 2013). For instance, $d d m 1$ mutant plants mainly lose repression of heterochromatic but not euchromatic TEs. To assess the location of the TE-dDHSs identified in the four comparisons, we plotted their genomic distribution against the relative density of genes and TEs (Fig. 4). Interestingly, the morc1/2-enhanced TE-dDHSs were preferentially associated with heterochromatin (Fig. 4, horizontal tracks 3 and 4), whereas the $P$. syringae pv. tomato-induced TE-dDHSs were more evenly distributed across the genome (Fig. 4, tracks 5 and 6). There is little, if any, overlap between the TE- or gene-associated dDHSs induced by $P$. syringae pv. tomato inoculation and those induced by the loss of MORC family members (Supplementary Fig. S3).

\section{DNase I-quantitative polymerase chain reaction (qPCR) analysis confirmed the chromatin accessibility of selected TE-dDHSs identified by DNase-Seq.}

Nine dDHSs associated with TEs, either in heterochromatin or in euchromatin, were randomly selected and were analyzed using DNase I qPCR to further assess the reproducibility of the DNase-Seq dataset. The $P$. syringae pv. tomato-inducible TEdDHS AP8539 was additionally chosen, as it mapped to a site proximal to the well-known defense gene $P R-1$ (Fig. 5A). This TE-dDHS spans a large region that encompasses two dDHSs, one resides in the promoter of a TE approximately $2 \mathrm{~kb}$ upstream of the $P R-1$ gene (designated as AP8539b) and the other is located within the $P R-1$ promoter (designated as AP8539a). Thus, we monitored chromatin accessibility at both sites. Using DNA prepared from DNase I-treated nuclei, qPCR was performed for the selected TE-dDHSs. Note that, while DNaseSeq identifies the ends of DNAs that are cut with DNase I, DNase I qPCR amplifies DNAs that are not disrupted by DNase I. Consequently, less amplification via qPCR corresponds to increased genome accessibility. Comparisons between the DNaseSeq reads and DNase I-qPCR analyses for morc1/2-enhanced TE-dDHSs (Fig. 5B) and for P. syringae pv. tomato-induced TEdDHSs (Fig. 5C) indicated that the results obtained from both

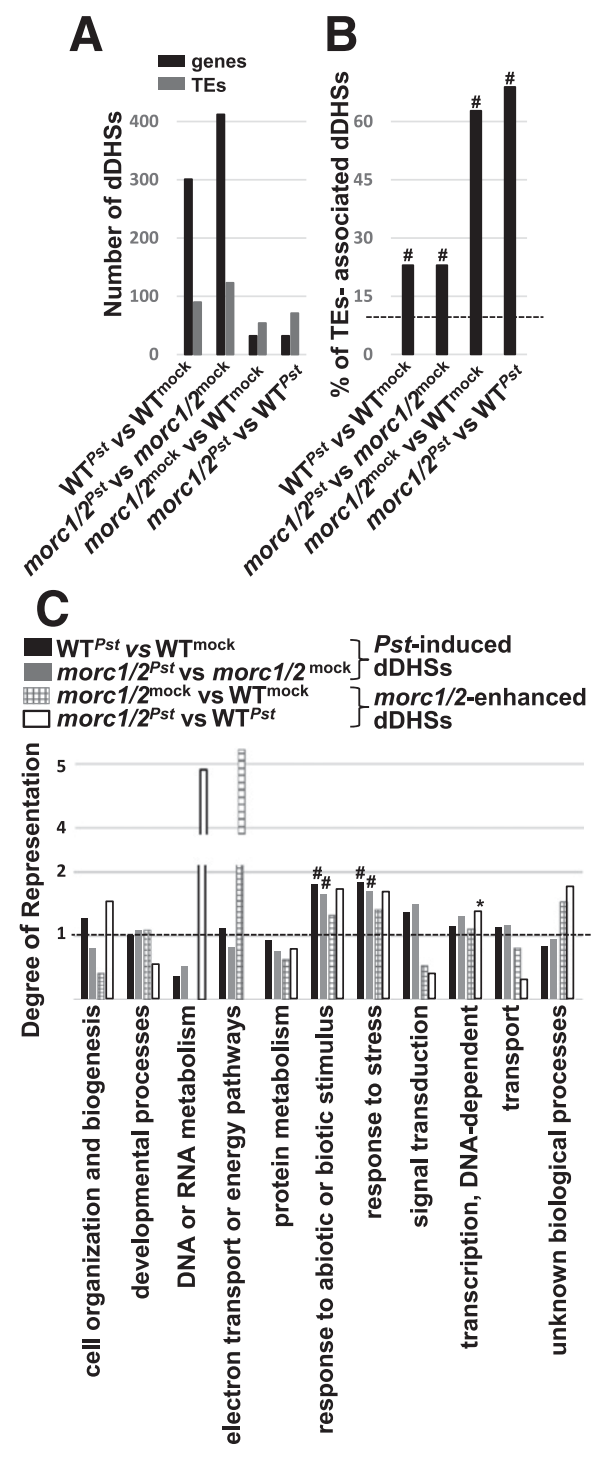

Fig. 3. Pathogen infection and mutations in MORC family members alter chromatin accessibility in transposable elements (TEs). A, The number of differential DNase I hypersensitive sites (dDHSs) present in the indicated pairwise comparisons were plotted. Genes and TEs are presented as black and gray bars, respectively. B, Percentage of TE-dDHSs. The broken black line indicates the percentage $(11.9 \%)$ of all TE-DHSs based on a total of 29,450 DHSs. C, Excluding TEs, the degree of representation in each biological process category was calculated for the dDHSs from each pairwise comparison, based on annotation in The Arabidopsis Information Resource database. Biological process categories are presented and their representation in the Arabidopsis genome is calculated at 1, denoted by a broken black line. Statistical significance was determined using a one sample $\chi^{2}$ test $(\mathrm{B})$ and the Benjamini-Hochberg procedure $(\mathrm{C})$; $*$ indicates $P<0.05$ and \# indicates $P<0.01$. 
techniques were consistent. The only exception was AP4254, which exhibited greater accessibility in morc $1 / 2$ plants than in WT plants following DNase-Seq but only a little difference between these plants following DNase I-qPCR.

Defense genes were overrepresented in the genomic regions proximal to $P$. syringae pv. tomato-induced TE-dDHSs and their induction by $P$. syringae pv. tomato was either delayed, reduced, or both in morc1/2.

Decreased methylation of some TEs in response to SA was shown to increase expression of neighboring genes (Dowen et al. 2012), suggesting that derepression of TEs might trigger the transcriptional activation of proximal genes. Therefore, annotated genes within $5 \mathrm{~kb}$ of TE-dDHSs were analyzed for their functional association (Fig. 6; Supplementary Table S8). Of the $P$. syringae pv. tomato-induced TE-dDHSs, a significant number were associated with genes involved in 'response to abiotic or biotic stimulus' or 'response to stress' (Fig. 6; Supplementary Table S9). A significant number of the morcl/2-enhanced TE-dDHSs were proximal to genes that are annotated as 'unknown biological processes'.

Of these TE-neighboring genes, those annotated as 'response to abiotic or biotic stimulus' included several well-known defense genes, such as $P R-1$ and $P R-2$. In addition, we selected 12 more defense genes that neighbor $P$. syringae pv. tomatoinduced TE-dDHSs and assessed their transcript levels at several time points after mock or virulent $P$. syringae pv. tomato infection. All of the genes analyzed displayed strong induction in response to infection, although their induction kinetics varied (Fig. 7). Although our analyses suggested that MORC proteins primarily associate with heterochromatic TEs, the previous demonstration that a wide range of TEs are upregulated in one or both morcl and morc6 (Moissiard et al. 2012), led us to test whether the defense genes neighboring $P$. syringae pv. tomato-induced

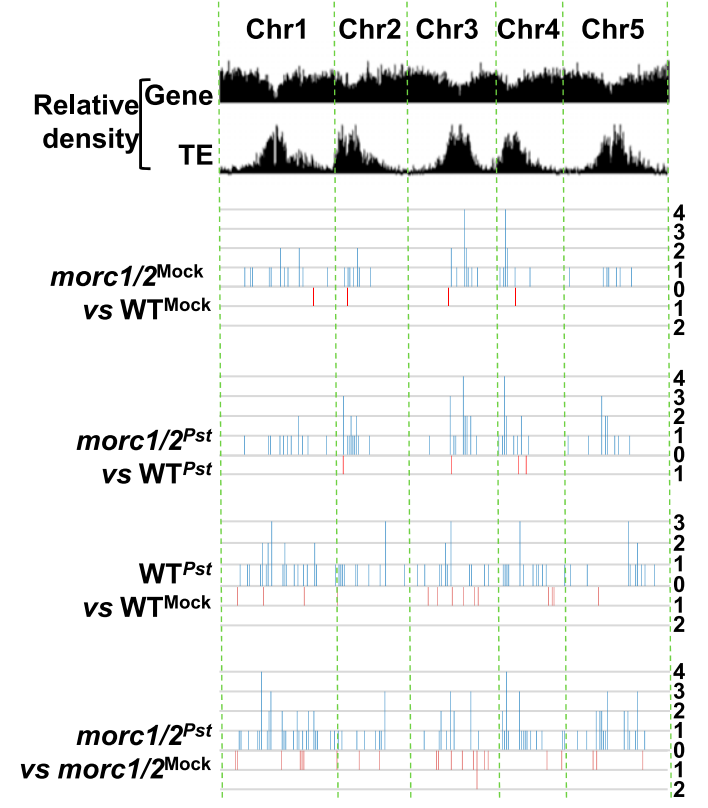

Fig. 4. Effects of MORC1 and infection on DNase I accessibility of heterochromatic transposable elements (TEs). The relative densities of genes and TEs are presented in the top two tracks and the genomic distribution of TE-associated differential DNase I hypersensitive sites (dDHSs) found in the four pairwise comparisons are shown in the lower four tracks. The $y$ axis indicates the number of dDHSs in a $100-\mathrm{kb}$ window. For each comparison, the DHSs enhanced in the plants listed first are presented in blue, while the DHSs enhanced in the plant listed second are presented in red. Dotted green lines indicate the borders between chromosomes.
TE-dDHSs exhibit altered expression in morc $1 / 2$. Notably, most of the 14 defense genes displayed various degrees of delayed or reduced transcriptional induction in $P$. syringae pv. tomatoinoculated $m o r c 1 / 2$ versus WT plants, suggesting that $\mathrm{MORC} 1 / 2$ promotes their expression. Note that, for four of the 14 genes (Atlg33960, Atlg80820, At2g20010, and At1g44350), the $P$. syringae pv. tomato-induced expression kinetics were not significantly different between morc $1 / 2$ and WT plants.

$P R-5$ is another extensively used marker gene for defense signaling. In contrast to $P R-1$ and $P R-2$, which have proximal $P$. syringae pv. tomato-induced TE-dDHSs, $P R-5$ has a $P$. syringae pv. tomato-induced dDHS within its own promoter and this location does not correlate with any annotated TEs. Nonetheless, $P R-5$ showed compromised induction by $P$. syringae pv. tomato in morc 1/2 as compared with WT (Fig. 7B). This observation prompted us to further assess whether the dDHS present in the $P R-5$ promoter is associated with a cryptic TE. Remarkably, four truncated putative TE sequences were observed at this dDHS (Supplementary Fig. S4). Together, these observations raise an intriguing possibility that TE-associated genomic regions help regulate gene expression in response to biotic stresses.

\section{$P$. syringae pv. tomato infection changed the profile of MORC1-TE interaction.}

Although we previously showed that MORC1 binds DNA with little sequence specificity in vitro (Kang et al. 2012), it is plausible that some of the morc1/2-enhanced TE-dDHSs are due to the loss of protection by bound MORC proteins. To address this possibility, chromatin immuno-precipitation followed by Illumina-based DNA sequencing (ChIP-Seq) was used to assess the interaction between Myc-tagged MORC1 and chromatin. These analyses were performed using an anti-Myc antibody and mock- or $P$. syringae pv. tomato-inoculated plants from a transgenic Arabidopsis morc1/2 line carrying a Myctagged MORC1 transgene expressed under its own promoter (Myc-gMORC1). This transgene was previously shown to complement morc $1 / 2$ (Kang et al. 2012). To control for nonspecific background binding, ChIP-Seq was performed in parallel on mock- and $P$. syringae pv. tomato-inoculated WT plants. Note that each sample was prepared in three independent biological triplicates.

Analysis of the ChIP-Seq peaks from mock- and P. syringae pv. tomato-inoculated Myc-gMORC1 plants (Fig. 8A, the third and fourth tracks; Supplementary Table S10) revealed that MORC1 binds sites distributed throughout the genome, although it shows a strong preference for heterochromatin. To find genomic regions exhibiting altered levels of MORC binding after pathogen infection, the intensity of ChIP-Seq peaks from mockand $P$. syringae pv. tomato-inoculated plants was compared. Differential ChIP-Seq peaks (dChIP peaks) exhibiting increased or decreased (Fig. 8A, induced and suppressed, blue lines and red lines, respectively, in the fifth track) intensity after $P$. syringae pv. tomato infection as compared with mock infection were not primarily associated with heterochromatic sites but, instead, were dispersed throughout the genome (Fig. 8A; Supplementary Table S11). Given that $P$. syringae pv. tomato-induced and morc1/2-enhanced dDHSs are enriched in TEs (Fig. 3), we assessed whether the genomic regions exhibiting altered interaction with MORC1 after pathogen infection correspond to TEs. Indeed, over $70 \%$ of the dChIP peaks that showed either increased or decreased MORC1 binding were TE-associated (Supplementary Fig. S5). Together, these results suggest that MORC1 is a chromatininteracting protein that displays a significant preference for TEs and exhibits altered binding affinity after pathogen infection. Interestingly, the MORC1-associated peaks identified by ChIPSeq were relatively broad, averaging around $8.5 \mathrm{~kb}$. This contrasts with the typically narrow peaks associated with transcription 
factors and suggests that MORC1 exhibits little sequence preference but, instead, may recognize higher-order chromatin structure.

Whether the MORC1-chromatin interaction sites overlap one or both the morcl/2-enhanced or $P$. syringae pv. tomatoinduced dDHSs was then assessed. All of the gene- and TEassociated dDHSs were checked for overlaps with the dChIP peaks; however, dDHSs located in the intergenic regions were excluded. The background level of overlap (Fig. 8B, red line) was determined by calculating the percentage of total gene- or TE-dDHSs in each group that overlap with $P$. syringae pv. tomato-suppressed or $P$. syringae pv. tomato-induced dChIP peaks. Interestingly, dChIP peaks that were reduced after $P$. syringae pv. tomato infection correlated most strongly with morc 1/2-enhanced TE-dDHSs, although a lower but statistically significant percentage overlapped with gene-associated dDHSs detected in morc $1 / 2$ versus WT plants after $P$. syringae pv. tomato infection. In comparison, the dChIP peaks that were enhanced by $P$. syringae pv. tomato infection showed a specific overlap with $P$. syringae pv. tomato-induced but not morc $1 / 2$-enhanced dDHSs located in genes. A low but statistically significant overlap between $P$. syringae pv. tomato-induced dChIP peaks and both $P$. syringae pv. tomato-induced and morcl/2-enhanced TE-dDHSs also was observed. Given the connection between

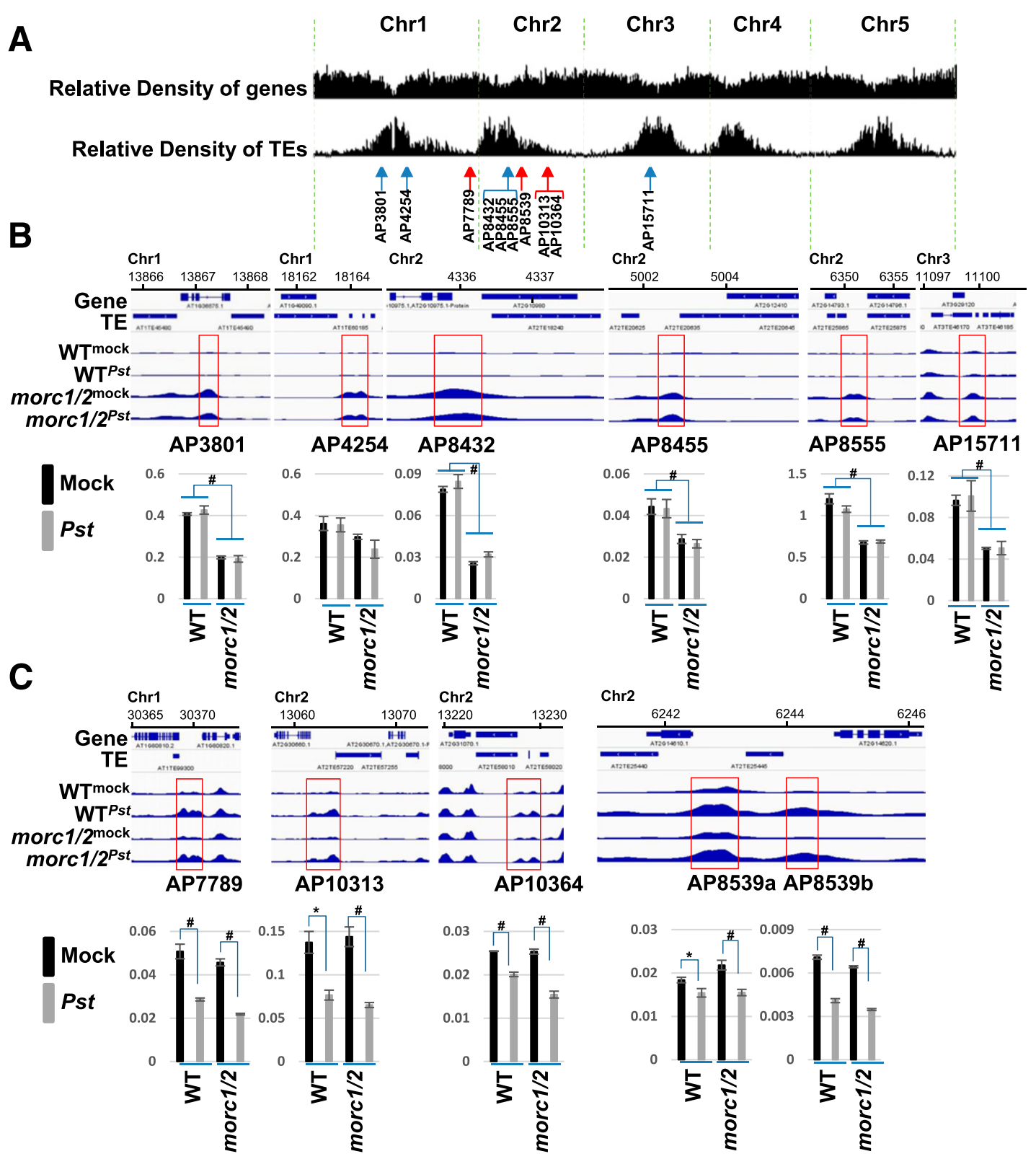

Fig. 5. Quantitative polymerase chain reaction (qPCR) analysis of selected differential DNase I hypersensitive sites (dDHSs) identified by DNase-Seq. A, Genomic locations of nine randomly selected transposable element (TE)-associated dDHSs as well as TE-associated dDHSs near the defense gene PR-1 (AP8539). dDHSs located in heterochromatic and euchromatic positions are indicated by blue and red arrows, respectively. A dotted green vertical line denotes the border between chromosomes. B and C, Schematic presentation of Arabidopsis genes and TEs, with their corresponding genome coordinates in kilobases, as well as DNase-Seq read densities of TE-associated dDHSs (indicated as red boxes) in mock- and Pseudomonas syringae pv. tomato (Pst)-inoculated wild

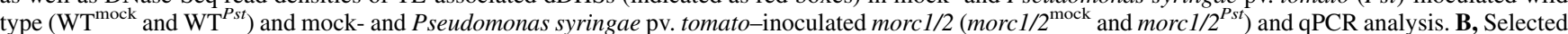
morc $1 / 2$-induced dDHSs. C, Selected $P$. syringae pv. tomato infection-induced dDHSs. The relative amount of each dDHS was determined through qPCR analysis using Tip41-like as a reference gene (represented in the $y$ axis). Note that DNase I-qPCR amplifies DNA that is not disrupted by DNase I; thus, lower levels of amplification indicate increased genome accessibility. Two repeats were performed for each dDHS. Statistical significance was determined using a Student's $t$ test; $*$ indicates $P<0.05$ and \# indicates $P<0.01$. 
MORC1 and TEs, we assessed whether cryptic TEs are present in the gene-dDHSs that overlap chromatin sites displaying increased MORC1 binding after P. syringae pv. tomato infection. Interestingly, cryptic TEs were identified in all of these genedDHSs (Supplementary Fig. S6), suggesting that MORC1 interacts with these previously unidentified TEs. In summary, our findings suggest that $P$. syringae pv. tomato infection leads to reduced MORC $1 / 2$ binding at dDHSs that are preferentially associated with heterochromatic TEs while, in contrast, infection enhances MORC1/2 binding at $P$. syringae pv. tomatoinduced dDHSs located in a small population of euchromatic and heterochromatic TEs as well as in genes, likely via unannotated cryptic TEs.

\section{Silencing of $P$. syringae pv. tomato-induced TE-dDHSs proximal to a MORC1 binding region compromised induction of adjacent defense genes.}

Although $\mathrm{MORC} 1 / 2$ physically associates with some $P$. syringae pv. tomato-induced TE-dDHSs (Fig. 8B), none of the TE-dDHSs neighboring the defense genes monitored in Figure 7 directly overlap $P$. syringae pv. tomato-induced dChIP peaks. However, a majority of these TE-dDHSs are within $250 \mathrm{~kb}$ of a $P$. syringae pv. tomato-induced dChIP peak (Supplementary Table S12). Based on these results, we hypothesized that, following $P$. syringae pv. tomato-induced binding of MORC1 at adjacent sites, these TE$\mathrm{dDHSs}$ are local enhancers that upregulate the expression of their neighboring defense genes. Suppression of these enhancers by

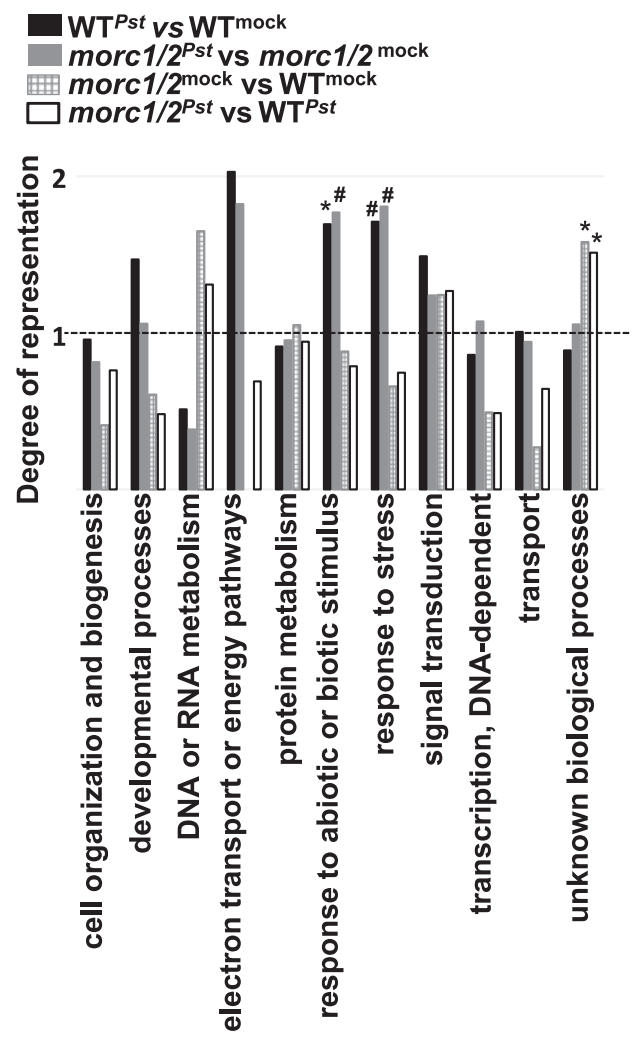

Fig. 6. The degree of representation of genes neighboring transposable element-differential DNase I hypersensitive sites (TE-dDHSs). Based on The Arabidopsis Information Resource annotation, biological process categories of the genes located within $5 \mathrm{~kb}$ of TE-dDHSs from each pairwise comparison were analyzed. The degree of representation of each category was plotted on the $y$ axis, as compared with their overall representation from all the genes in the Arabidopsis genome, which was set at 1 and denoted by a broken line. Statistical significance was determined using the Benjamini-Hochberg procedure; * indicates $P<0.05$ and \# indicates $P<$ 0.01 .
RNAi-mediated silencing would, therefore, be expected to interfere with proximal gene induction. To test this hypothesis, transgenic Arabidopsis lines expressing a hairpin construct (Wesley et al. 2001) targeting each of seven TE-dDHSs shown in Figure 7 were generated. Five of these TE-dDHSs were located within $250 \mathrm{~kb}$ of one or more infection-induced dChIP peak; the other two dDHSs that were not proximal to a dChIP peak were chosen as controls. Silencing of the targeted regions was assessed via $M c r B C-\mathrm{qPCR}$, which involves qPCR of DNA digested with or without $M c r B C$, an enzyme that specifically cuts methylated DNA. This quantitative analysis to test whether DNA methylation is induced by a hairpin RNA verified that all seven lines have significant DNA methylation in the intended regions (Fig. 9A). Silencing the putative TEassociated enhancers proximal to a $P$. syringae $\mathrm{pv}$. tomato-induced $\mathrm{dChIP}$ peak significantly reduced the induction of four of the five neighboring defense genes, including $P R-1, A t 2 g 06050$, At4g 19230, and At $2 g 27690$ but did not impact At $3 g 44300$ (Fig. 9B). By contrast, silencing the TE-dDHSs lacking an adjacent $P$. syringae pv. tomato-induced dChIP peak had little impact on the induction of defense genes At2g19230 and At4g16760 (Fig. 9B). These results support the possibility that these MORC1associated TEs are local enhancers that induce adjacent defense gene expression.

\section{DISCUSSION}

To address the relationship between the roles MORC proteins play in gene silencing and plant immunity, we mapped the genomic location of DHSs in mock- and pathogen-inoculated WT and MORC-deficient plants. In a previous study, approximately $45 \%$ of the DHSs in the Arabidopsis genome were mapped to putative promoter regions (Zhang et al. 2012). Underscoring the correlation between DHSs and transcriptionally active chromatin, these sites were depleted of nucleosomes and were tightly associated with RNAPII binding sites. Furthermore, the prevalence of DHSs was dramatically reduced in transcriptionally silent pericentromeric regions that contain highly methylated DNA. Similar to these findings, the majority of DHSs we identified were located within $1 \mathrm{~kb}$ upstream of the TSS of protein-coding genes. Analysis of the TE-DHSs also revealed that the majority also were located within $1 \mathrm{~kb}$ of the TSS. Neither P. syringae pv. tomato infection nor loss of MORC family members substantially altered the genomic distribution of DHSs. Given that DNase I sensitivity is influenced by DNA methylation levels (Zhang et al. 2012) and that genome-wide DNA methylation levels are not appreciably altered in morcl or morc6 mutants as compared with WT plants (Moissiard et al. 2012) or in P. syringae pv. tomato- versus mock-inoculated Arabidopsis (Dowen et al. 2012), our DHS results are consistent with these methylation studies.

Although global DHS distribution was not affected by P. syringae pv. tomato inoculation or the loss of MORC family members, pairwise comparisons of the DHSs detected in mockor $P$. syringae pv. tomato-inoculated WT or morc1/2 plants revealed notable differences. To our surprise, however, little difference was observed in dDHSs between virulent and avirulent $P$. syringae pv. tomato (Fig. 2A), while considerably more $\mathrm{dDHSs}$ were found for $P$. syringae pv. tomato versus mock than for avirulent $P$. syringae pv. tomato versus mock, suggesting that infection with virulent $P$. syringae pv. tomato alters genome accessibility more than avirulent $P$. syringae pv. tomato infection. However, since host responses to avirulent and virulent infection are often quantitative (Tao et al. 2003) and the analysis was done with only one time point, additional time points will likely be required to assess the quantitative nature of avirulent versus virulent infection-induced genome accessibility. It is noteworthy that a large number of dDHSs found both in a $P$. syringae pv. tomato versus mock and in an avirulent $P$. syringae 
pv. tomato versus mock comparisons, when the DHSs were compared between these virulent and avirulent $P$. syringae pv. tomato, the differences in their DHSs were marginal.

The number of morcl/2-enhanced dDHSs was much lower than that induced by $P$. syringae pv. tomato infection (Fig. 3). Of these morc 1/2-enhanced dDHSs, fewer than $40 \%$ were located in genes. Instead, morc $1 / 2$-enhanced dDHSs were highly enriched in TEs, particularly those located in heterochromatic regions (Fig. 4). Analysis of the genomic regions identified by ChIP-Seq confirmed that MORC1 preferentially binds heterochromatic and TE-associated regions (Fig. 8A). Furthermore, since morc $1 / 2$-enhanced TE-dDHSs overlap the genomic regions corresponding to $P$. syringae $\mathrm{pv}$. tomato-suppressed dChIP peaks at a rate substantially greater than that of the expected background, $P$. syringae pv. tomato infection appears to lead to lowered MORC1/2 binding at heterochromatic
TEs (Fig. 8B). It is interesting to note that a majority of the TEs transcriptionally activated in morc 1 , morc6, and morc $1 / 6$ (Moissiard et al. 2012) are physically located close (less than $160 \mathrm{~kb}$ ) to morcl/2-enhanced TE-dDHSs. Together, these results are consistent with the reported association of MORC1 and MORC6 with heterochromatin (Moissiard et al. 2012) and the derepression of multiple families of TEs and endogenous genes preferentially associated with heterochromatin in one or more morc1, morc2, or morc6 mutants (Moissiard et al. 2012; Brabbs et al. 2013; Moissiard et al. 2014).

Given the considerable interaction between MORC1 and heterochromatin (Fig. 8A), it seems surprising that dramatic differences in chromatin accessibility, as determined by the number of dDHSs, were not observed in comparisons between morc $1 / 2$ and WT plants receiving the same mock or $P$. syringae pv. tomato treatment (Figs. 2 and 3). One possible explanation

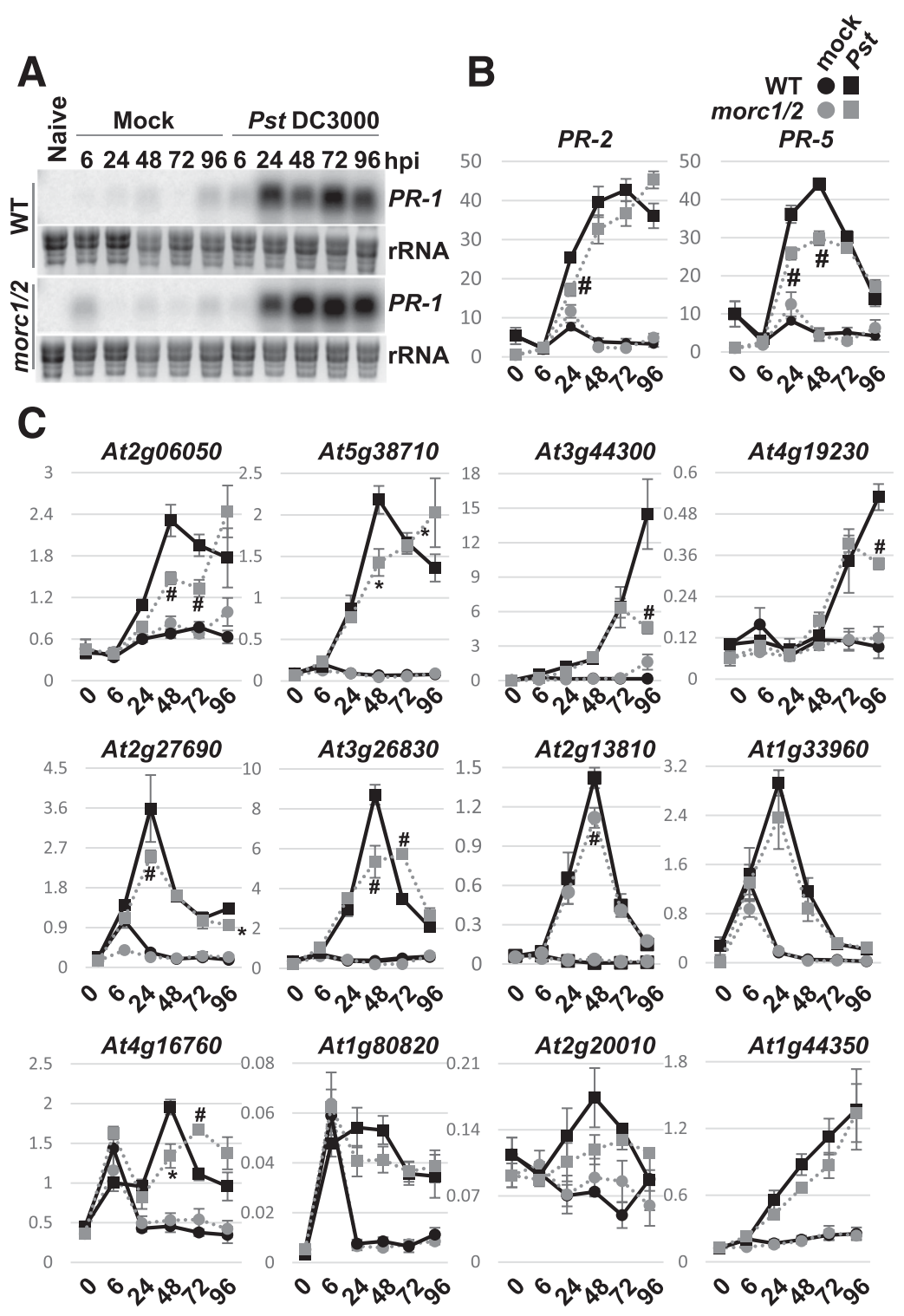

Fig. 7. MORC1/2 influences one or both the kinetics or amplitude of defense genes induced by Pseudomonas syringae pv. tomato $(P s t)$. A, $P R-1, \mathbf{B}, P R-2, P R-5$ and $\mathbf{C}$, selected defense genes that neighbor transposable element (TE)-associated differential DNase I hypersensitive sites were analyzed. Leaves were inoculated with buffer (mock) or $10^{6} \mathrm{CFU}$ of $P$. syringae pv. tomato per milliliter. Untreated (naïve) control leaves were harvested at $0 \mathrm{~h}$ postinoculation (hpi). RNAs prepared from wild type (WT) and morc $1 / 2$ plants at the indicated time points after inoculation were resolved in a $1.5 \%$ agarose gel followed by Northern blot analysis with a probe for $P R-1$ (A) and were used for quantitative reverse transcription-polymerase chain reaction (qRT-PCR) analysis with primers specific for indicated genes (B and C). Ribosomal RNA was used as a loading control for Northern blot (A). The TIP41-like gene was used as a reference gene for qRT-PCR and the mean \pm standard error $(n \geq 6)$ including a minimum of two biological replicates is presented (B and C). Statistical significance from WT was determined using $t$ test; * indicates $P<0.05$ and \# indicates $P<0.01$. 
for the discrepancy in these findings is that the development of DHSs in the morcl/2 background may be partially suppressed by one or more functionally redundant members of the MORC family. Indeed, MORC3, the next closest homolog of MORC1 after MORC2, appears to be functionally redundant with MORC1 to some degree, since it restored TCV coat proteininduced cell death in morcl-1 plants expressing an inducible coat protein transgene (Kang et al. 2008) (Supplementary Fig. S7). Unfortunately, we cannot test whether MORC3 also suppresses DHS development in the morc $1 / 2$ background, because the morc3-1 knock-out mutation (SALK_000009) is lethal in the homozygous state (Kang et al. 2010).

An alternative possibility is that MORC1/2 does not directly bind DNA but, instead, influences chromatin accessibility by directly or indirectly interacting with other proteins. For example, MORC family members may influence DNase I hypersensitivity in heterochromatic regions by interacting with proteins involved in RdDM. Indeed, the combined observations
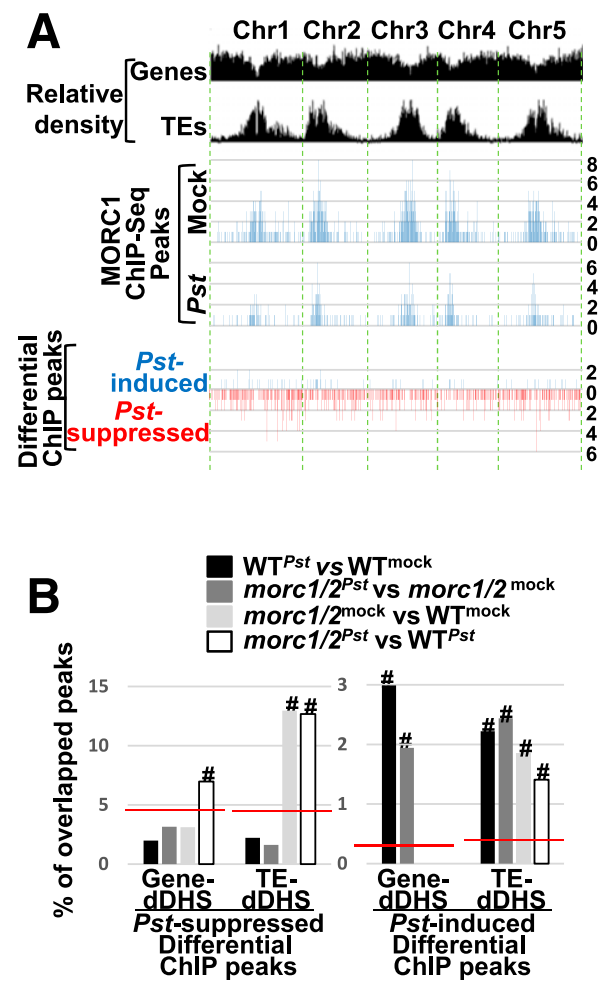

Fig. 8. MORC1 is physically associated with infection-induced transposable elements-differential DNase I hypersensitive sites (TE-dDHSs). MycgMORC1 plants and wild type (WT) were mock-or $P$. syringae pv. tomato $(P s t)$-infected $\left(5 \times 10^{5} \mathrm{CFU} / \mathrm{ml}\right)$ for 1 day. Chromatin immunoprecipitation (ChIP) was performed using an anti-Myc antibody, and the recovered DNAs were sequenced, using Illumina Hi-Seq 2500. SICER (Zang et al. 2009) was used to identify peaks that are associated with MORC1 in three independent biological replicates; WT was used as a background control. A, The relative densities of genes and TEs are presented in the top two tracks. The genome position of ChIP-Seq peaks $(P<$ 0.01 ) in mock- and $P$. syringae pv. tomato-infected Myc-gMORCl plants as compared with the WT background control are presented in the third and fourth tracks. The $y$ axis indicates the number of ChIP peaks in a 100-kb window. ChIP-Seq peaks (false discovery rate $<0.001$ ) for which levels are substantially different in mock- versus $P$. syringae pv. tomato-inoculated plants (termed differential ChIP peaks) are presented in the fifth track. B, Percentage of dDHS peaks overlapping with ChIP peaks were calculated to show association of MORC1 with dDHSs. To better display TE-association with MORC1, gene- and TE-dDHSs were separately analyzed. A background level of overlap between ChIP and dDHS peaks was calculated using total gene- $(n=24,340)$ and TE-DHSs $(n=3,520)$. Statistically significant overrepresentation was determined between indicated groups and all DHSs using one sample $\chi^{2}$ test; \# indicates $P<0.01$. that i) derepression of reporter genes in morc6 mutants correlates with a decrease in their DNA and histone methylation levels (Brabbs et al. 2013; Lorković et al. 2012) and ii) MORC1, MORC2, MORC6, singly or in combination, interact with several proteins involved in the RdDM pathway, including

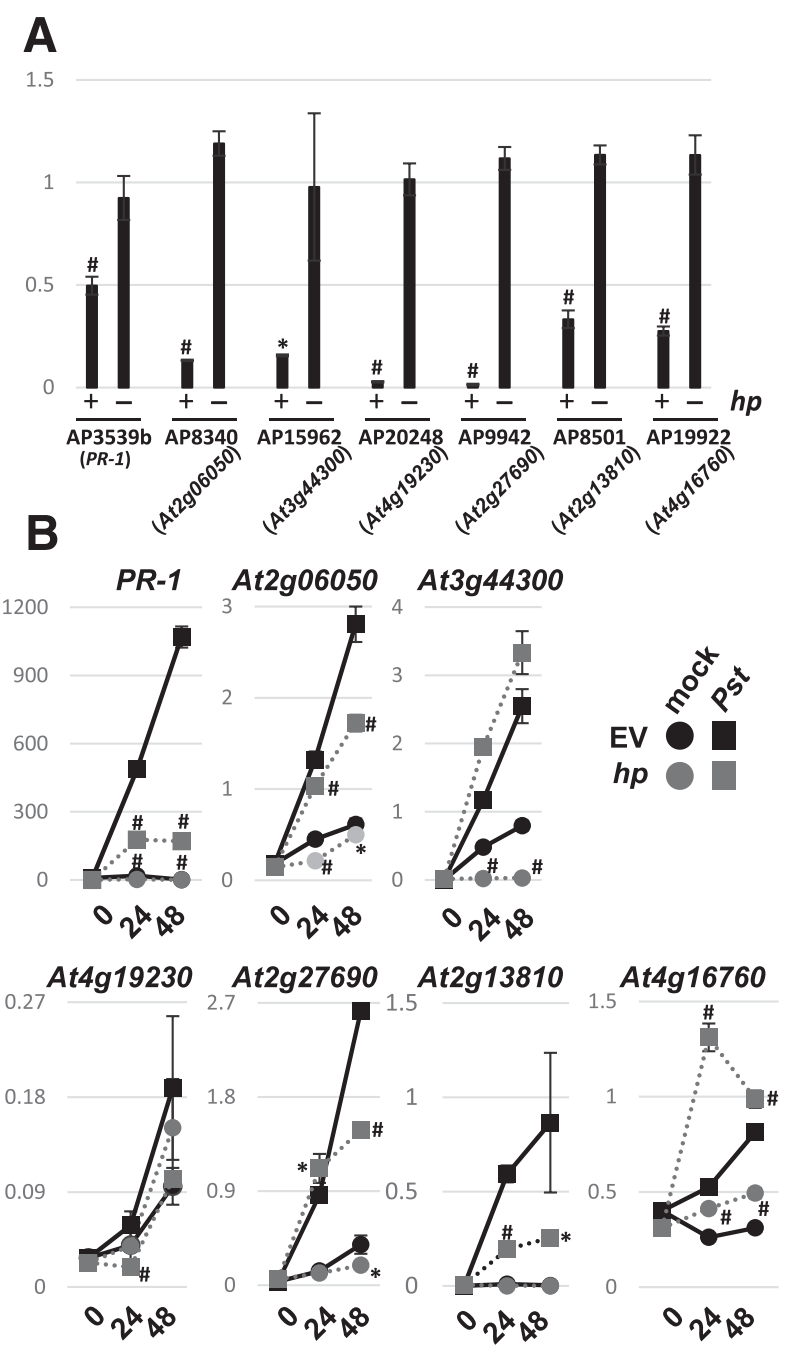

Fig. 9. Silencing of Pseudomonas syringae pv. tomato-induced transposable elements-differential DNase I hypersensitive sites (TE-dDHSs) proximal to a MORC1 binding site compromised induction of adjacent defense genes. A, RNA interference-mediated silencing of selected TEdDHSs in transgenic Arabidopsis plants carrying a hairpin construct $(h p)$ was verified via $M c r B C$-qPCR (quantitative polymerase chain reaction); wild type (WT) plants were used as a control. The relative amount of $M c r B C$-digested DNA in comparison with undigested DNA was analyzed in three replicates and is presented in the $y$ axis. Note that higher levels of amplification indicate decreased DNA methylation. Several independent lines were generated for each hairpin construct. B, P. syringae pv. tomato-induced expression of defense genes proximal to silenced TEassociated dDHSs was analyzed via quantitative reverse transcription (qRT)-PCR. Each panel corresponds to a representative transgenic line $(h p)$ in which the TE-dDHS adjacent to the indicated defense gene has been silenced by a hairpin construct; an empty vector (EV)-transformed line was used as a control. Leaves were inoculated with buffer (mock) or $10^{6} \mathrm{CFU}$ of $P$. syringae pv. tomato per milliliter. Untreated (naive) control leaves were harvested at $0 \mathrm{~h}$ postinoculation. RNAs prepared from WT and morc $1 / 2$ plants at the indicated time points after inoculation were used for qRT-PCR analysis with primers specific for indicated genes. The TIP41-like gene was used as a reference gene for qRT-PCR and the mean \pm standard error $(n \geq 3)$. A minimum of one more independent line was tested and found to have an expression pattern comparable to those presented here. Statistical significance from WT was determined using $t$ test; * indicates $P<0.05$ and \# indicates $P<0.01$. 
DMS3 (defective in meristem silencing 3) (Lorković et al. 2012) and the SET domain-containing proteins SUVH9 and SUVH2 (Liu et al. 2014), suggest that MORC family members influence RdDM, which in turn could affect DNase I sensitivity. The relationship between MORC proteins and RdDM, however, is currently unclear. Notably, older morc6 plants develop stochastic, cell-autonomous silencing of a green fluorescent protein (GFP) reporter gene that is consistently expressed in younger plants (Brabbs et al. 2013). Since this silencing of GFP expression was associated with the reappearance of DNA methylation in the reporter gene sequence, it was proposed that MORC6 promotes but is not obligately required for RdDM (Brabbs et al. 2013). Consistent with this proposition, analyses of morcl and morc6 mutants failed to detect a correlation between changes in genomewide methylation levels and the activation of a silenced reporter gene (Moissiard et al. 2012, 2014). Instead, since pericentromeric heterochromatin was decondensed in these mutants, it was proposed that MORC1 and MORC6 enforce the higher order compaction of methylated, silenced chromatin; such a function would, presumably, also modulate the DNase I sensitivity of these sequences. The very broad MORC1-associated peaks identified by our ChIP-Seq analysis also support this possibility.

In comparison with the morcl/2-enhanced dDHSs, those induced by $P$. syringae pv. tomato infection were primarily located in protein-encoding genes, particularly those associated with biotic or abiotic stresses. They also were enriched in TEs; these TE-dDHSs were spread throughout the genome and shared little overlap with those enhanced by morcl/2 (Fig. 4). Strikingly, the 5' flanking regions of a wide range of defense genes, including $P R-1$, $P R-2$, and $P R-5$, contained $P$. syringae pv. tomato-induced TE$\mathrm{dDHSs}$. Since the expression kinetics of these $P R$ genes as well as 12 additional, randomly selected defense genes was generally modestly delayed or weaker in $P$. syringae pv. tomato-inoculated morcl/2 as compared with WT plants (Fig. 7), MORC1/2 appears to promote their expression. Consistent with this possibility, MORC1 association with $P$. syringae pv. tomato-induced TE$\mathrm{dDHSs}$ was enhanced after $P$. syringae pv. tomato infection. Note that all of the $P$. syringae pv. tomato-induced gene dDHSs were found to be associated with unannotated TEs, essentially making them TE-dDHSs (Fig. 8B). It is interesting to note that infectioninduced transcription of $P R-1$ and other defense genes was generally suppressed by RNAi-silencing of the neighboring (although in some cases distant) TE-dDHS but only if the TE-dDHS was adjacent to a $P$. syringae pv. tomato-induced dChIP peak. Given that enhancers can be located as far as $1 \mathrm{Mb}$ from the genes they regulate (Krivega and Dean 2012; Yang and Corces 2012), our silencing results suggest that i) at least some $P$. syringae $\mathrm{pv}$. tomato-induced TE-dDHSs serve as enhancers of neighboring defense genes and ii) these putative enhancers are activated by $P$. syringae $\mathrm{pv}$. tomato-induced MORC1/2 binding to sites that, while nearby, do not necessarily overlap with the dDHS. This latter conclusion may explain why the overlap frequency between dDHSs and ChIP peaks is relatively low (Fig. 8B). Moreover, this long-distance effect of MORC1 recruitment on defense genes underscores upcoming challenges in characterizing chromatin-interacting proteins such as MORC1 and its homologs in the modulation of associated genes. It also remains to be seen why MORC1/2 influenced defense genes in a limited fashion rather than globally and how this observation is related to the earlier report showing the stochastic silencing phenotype of a morc6 mutant (Brabbs et al. 2013).

Analyses of the genomic regions exhibiting differentially increased or decreased MORC1 binding following P. syringae pv. tomato infection indicated that they were strongly associated with TEs dispersed over the entire genome (Fig. 8A). This finding, combined with the discovery that cryptic TEs are present in most of the gene-associated dDHSs exhibiting increased MORC1 binding after $P$. syringae pv. tomato infection, suggests that MORC1 may regulate gene expression by binding the super structure associated with TEs or TE-like sequences. A long list of studies also have suggested a link between TEs and gene regulation. In humans, TEs and repeat DNAs (Feschotte 2008) are associated with cell-specific transcription (Thurman et al. 2012). Furthermore, a substantial portion of regulatory elements in the promoters of human and plant genes are derived from TEs or pseudo or former TEs (Girard and Freeling 1999; Jordan et al. 2003; Martienssen et al. 1990). In Arabidopsis, TE density is enriched in the approximately $2 \mathrm{~kb}$ upstream of the TSS and near the $3^{\prime}$ end of genes whose induction by SA or P. syringae pv. tomato treatment is associated with the appearance of differentially methylated regions (Dowen et al. 2012). Analysis of these P. syringae pv. tomato- or SA-inducible genes containing differentially methylated regions revealed that many exhibit functions associated with plant immunity. A recent study also indicated that DNA demethylation of TEs is important for the activation of some defense genes (Le et al. 2014). Our finding that $P$. syringae pv. tomatoinduced TE-dDHSs are associated with a wide range of defense genes further argues that TEs are important regulatory elements for controlling transcription and that their modulation by RdDM components, including MORC1, plays an important role in the activation of defense responses after pathogen attack.

In summary, we demonstrate that $P$. syringae pv. tomato infection primarily suppresses MORC1 binding at dDHSs associated with heterochromatic TEs but enhances its binding at infection-induced dDHSs in genes and TEs. These results, combined with MORC1's previously demonstrated involvement in heterochromatin condensation and gene silencing (Brabbs et al. 2013; Lorković et al. 2012; Moissiard et al. 2012), and our finding that defense gene expression is attenuated in the morcl/2 mutant, suggest that MORC1 plays important roles in both gene silencing and gene induction. We propose that MORC1 mediates these divergent effects via its interaction with different chromatin-binding proteins. In this scenario, the $P$. syringae pv. tomato-induced loss of MORC1 at heterochromatic TEs would disrupt a complex involved in gene silencing, thereby leading to activation of TE expression after pathogen infection. By contrast, the $P$. syringae pv. tomato-induced addition of MORC1 to a protein complex present at TEs would temporarily relieve silencing, thereby promoting robust expression of proximal genes. In support of this model, growing evidence suggests that epigenetic regulation is often context specific (Sarris et al. 2014). For instance, enhancer of Zeste 2 homolog (EZH2), which is a core enzymatic subunit of the polycomb repressive complex 2 (PRC2) in human cells, exerts opposing effects on gene expression. As a part of PRC2, EZH2 is involved in silencing a wide range of genes; however, when EZH2 is posttranslationally modified, it functions as a coactivator for androgen receptor-target genes (Xu et al. 2012). Further characterization of MORC1's interacting proteins, including epigenetic factors such as DMS3 (Lorković et al. 2012), SUVH9, and SUVH2 (Liu et al. 2014), will likely provide important insights into the mechanisms through which MORC1 impacts gene silencing, defense gene induction, and plant immunity.

\section{MATERIALS AND METHODS}

\section{Plant material, generation of transgenic lines, bacterial infiltration, and analysis of antibacterial resistance in Arabidopsis.}

Plants were grown in soil at $22^{\circ} \mathrm{C}, 60 \%$ relative humidity, and a 16-h light period. Plants 3.5 weeks old were syringeinfiltrated with $P$. syringae pv. tomato $\left(5 \times 10^{5} \mathrm{CFU} / \mathrm{ml}\right)$ or $10 \mathrm{mM} \mathrm{MgCl} 2$ (mock) and treated leaves were harvested 1 day postinoculation for DNase-Seq, DNase-qPCR, and ChIP-Seq 
described below or at the indicated times for RNA analysis. Transgenic Arabidopsis lines were generated using a floral dipping method as previously described (Kang et al. 2008). To analyze antibacterial resistance in Arabidopsis, leaves of Arabidopsis plants were infiltrated with $P$. syringae pv. tomato with or without AvrRpt2 at a rate of $10^{5} \mathrm{CFU} / \mathrm{ml}$ in $10 \mathrm{~mm} \mathrm{MgCl}_{2}$, using a needleless syringe. Infected leaves were harvested at the given time points after the infiltration, and then, were used for bacterial titer determination, as previously described (Kang et al. 2008).

\section{Preparation for DNase-Seq and DNase-qPCR.}

Around $4.5 \mathrm{~g}$ of leaf tissue was harvested in a $50 \mathrm{ml}$ tube and was incubated in diethyl ether for $3 \mathrm{~min}$, followed by washing three times with cold water. The tissue was homogenized in $5 \mathrm{ml}$ of the homogenization buffer at $4^{\circ} \mathrm{C}$, using a T10 UltraTurrax homogenizer (IKA). Nuclei were enriched as previously described (Manzara and Gruissem 1995). In addition, the nucleus pellet was washed five times with the homogenization buffer to remove chloroplasts and $P$. syringae pv. tomato, followed by a Percoll gradient purification as described (Henfrey and Slater 1988).

Prepared nuclei were subject to DNase I digestion as described (Hesselberth et al. 2009), with the following modification: After concentration of nuclei was calculated using a hemocytometer under the microscope, $8 \times 10^{5}$ nuclei were incubated with one unit of DNase I (Roche) at $37^{\circ} \mathrm{C}$ for $10 \mathrm{~min}$, which gave DNA cleavage comparable to that in the yeast DNase-Seq experiment (Hesselberth et al. 2009). DNA was size-fractionated, using a sucrose gradient as described (Hesselberth et al. 2009), to obtain DNA ranging from 100 to $700 \mathrm{bp}$. DNA was further purified using a Qiagen PCR purification kit. DNA concentration was determined using PicoGreen (Life Technologies), and DNA quality was checked on a $1 \%$ agarose gel stained with SYBR green (Life Technologies) and scanned with a Typhoon Trio imager (GE Healthcare).

\section{Construction and sequencing of DNase I libraries.}

Each combination of genotype and treatment was prepared in biological triplicates, resulting in a total of 36 independent libraries. DNase I libraries were prepared using a Genomic DNA sample prep kit (Illumina) according to the manufacturer's protocol. The libraries were sequenced on an Illumina Hiseq 2500 system at Cornell University Life Sciences Core Laboratories Center with single-end, $100 \mathrm{bp}$ mode.

\section{DNase-Seq data and gene ontology analysis.}

DNase-Seq reads were first aligned to the Arabidopsis chloroplast genome using Bowtie (Langmead et al. 2009), allowing up to two mismatches, and those aligned were discarded. The resulting reads were then aligned to the Arabidopsis genome (TAIR10 release) using Bowtie, allowing up to two mismatches, and only the best hits were kept. Only reads uniquely mapped (having one single best hit) to the genome were used for further analysis. The uniquely mapped reads from biological replicates were combined, and their mapping information was then fed to F-seq (Boyle et al. 2008) to identify DHSs with default parameters. The identified DHSs from different samples that overlapped were merged into one DHS. If the peak position was covered by more than one type of genomic feature, the annotation was determined by a predefined order. The order of genomic features was i) TSS upstream, ii) TE upstream, iii) UTR, iv) exon or intron, v) TE, vi) TTS downstream, vii) TE downstream, and viii) intergenic. Then for each DNase-Seq library, the number of reads mapped to each of the identified DHSs was counted and normalized to reads per million mapped reads. The raw count information was fed to DESeq (Anders and Huber 2010) to identify differentially accessible DHSs upon infection by $P$. syringae pv. tomato in
WT and MORC-deficient mutants with a cutoff of corrected $P$ value $<0.05$ and fold change $>2$.

To evaluate enrichment in the gene ontology categories, known or deduced biological functions of the genes associated with dDHSs were annotated with TAIR (Berardini et al. 2004) as of January 2015. The broad biological categories 'other biological processes,' 'other cellular processes,' and 'other metabolic processes' were not presented in Figures 3 and 6 . The raw $P$ values were calculated using the hypergeometric distribution, which were then adjusted for multiple testings using the Benjamini-Hochberg procedure (Benjamini and Hochberg 1995).

\section{ChIP.}

ChIP was performed as described (Tai et al. 2005) with the following changes: $4.5 \mathrm{~g}$ of leaf tissue or sample was crosslinked for $60 \mathrm{~min}$ and nuclei were prepared as described above, except that the Percoll gradient step was skipped. Magnetic beads conjugated with protein A/G (Thermo Fisher Scientific) and $\alpha$-myc (Abcam) antibodies were used to immunoprecipitate Myc-tagged MORC1 and its interacting chromatin. DNA was purified using the Qiagen PCR purification kit. ChIP-Seq libraries were generated using the NEBNext ChIP-Seq library prep reagent kit (New England Biolabs) according to the manufacturer's instructions. The libraries were sequenced using an Illumina Hiseq 2500 system at the Genomic Sequencing and Analysis Facility of The University of Texas at Austin.

\section{ChIP-Seq data analysis.}

ChIP-Seq raw reads were first processed to remove adapter and low-quality sequences using Trimmomatic (Bolger et al. 2014). The statistical elements of this analysis are shown in Supplementary Table S13 and Supplementary Table S14. For each library, identical reads were then collapsed into a single unique read. The resulting ChIP-Seq reads were aligned to the Arabidopsis genome (TAIR10 release) using Bowtie (Langmead et al. 2009), allowing up to one mismatch, and only the best hits were kept and only reads uniquely mapped (having one single best hit) to the genome were kept. The alignments from different biological replicates were combined using SAMtools ( $\mathrm{Li}$ et al. 2009), and then were converted into browser extensible data (BED) format using BEDtools (Quinlan and Hall 2010). The read mapping coordinates in BED format were fed into SICER (Zang et al. 2009) (redundancy threshold at 1 , window size at 600 , fragment size at 150 , effective genome fraction at 0.96 , and gap size at 1,800 .) to identify wide peaks from the two pairs of libraries, i.e., i) $M y c-g M O R C 1^{\text {mock }}$ and $\mathrm{WT}^{\text {mock }}$ and ii) $M y c-g M O R C 1^{P s t}$ and $\mathrm{WT}^{P S t}$. SICER were run at the following parameters: redundancy threshold at 1 , window size at 600 , fragment size at 150 , effective genome fraction at 0.96 , and gap size at 1,800 . SICER first identified significant peaks in each of the two pairs and, then, merged the two sets of peaks. Then for each merged peak, its level in P. syringae pv. tomato was compared with that in mock to determine the significance of changes. Significantly differential peaks were identified when the fold change of peak levels between $P$. syringae pv. tomato and mock should be larger than 1.1 and the false discovery rate should be less than 0.001 .

Then for each ChIP-Seq peak, the number of mapped reads in each sample was counted and normalized to reads per kilobase per million mapped reads. Raw counts were then fed to edgeR (Robinson et al. 2010) to identify differential peaks between WT and Myc-gMORC1 plants under mock or P. syringae pv. tomato inoculation. ChIP-Seq peaks with fold changes of peak levels $>1.1$ and $P$ value $<0.01$ were identified as significant and MORC1-associated. 


\section{qPCR.}

qPCR was used to quantify the DNA templates prepared from DNase I and ChIP experiments. Maxima SYBR green qPCR master mix (Thermo Fisher Scientific) was used, with initial incubation at $50^{\circ} \mathrm{C}$ for $2 \mathrm{~min}$ and at $95^{\circ} \mathrm{C}$ for $10 \mathrm{~min}$, followed by 40 cycles of $95^{\circ} \mathrm{C}$ for $25 \mathrm{~s}$ and $60^{\circ} \mathrm{C}$ for $1 \mathrm{~min}$. The primer set used for qPCR was listed in Supplementary Table S15. Level of target DNA was calculated from the difference of threshold cycle values between reference and target genes (Schmittgen and Livak 2008). The TIP41-like gene (Czechowski et al. 2005) was used as the reference for DNase I-qPCR.

\section{Northern blot and qRT-PCR analysis.}

RNA was extracted using TRIzol (Life Technologies) following the manufacturer's protocol and was quantified by NanoDrop 1000 (Thermo Fisher Scientific). Northern analysis was performed with the $P R-1$ probe as described (Kang and Klessig 2005) because $P R-1$ gene has no introns. Therefore, the PCR product made off the cDNA is not distinguishable from the PCR product produced off any small amount of contaminating genomic DNA, which could obscure real but subtle differences in expression levels (Fig. 7A). qRT-PCR analysis was performed as described (Kang et al. 2010); the TIP41-like gene (Czechowski et al. 2005) was used as a reference gene.

\section{McrBC-qPCR.}

Overnight digestion of $500 \mathrm{ng}$ of genomic DNA was performed with and without $M c r B C$ as suggested by the manufacturer (New England Biolabs). The digested sample was diluted 10 -fold and was used for qPCR, which is described above.

\section{DNA constructs for complementation and RNAi silencing.}

Constructs complementing morc $1-1$ were previously described (Kang et al. 2008). NheI-digested bacterial artificial chromosome DNA F23E13 carrying genomic MORC3 was cloned into pART27. This construct was used for the complementation of morc 1-1. Hairpin constructs for RNAi silencing were generated as described (Kang et al. 2003), using pHannibal (Wesley et al. 2001). The binary vector pART 27 carrying the NotI cassette from pHannibal was then transformed into Arabidopsis.

\section{Accession number.}

Raw DNase-Seq and ChIP-Seq reads have been deposited in the National Center for Biotechnology Information sequence read archive under accession number SRP055733.

\section{ACKNOWLEDGMENTS}

We thank D. Dempsey for critical comments on the manuscript and E. A. Kim, N. Dharmasiri, D. Hahn, R. Walter, S. Baek, D. H. Kim, E. Kim, and S. Sung for technical support. We thank J. A. Stamatoyannopoulos for sharing detailed protocols for DNase-Seq and S. Jacobsen for morc6 mutants. This work is supported by the Texas State University Faculty Startup Program and Multi-disciplinary Internal Research grant to H. G. Kang, and National Science Foundation grants (IOS-1553613 to H.-G. Kang and IOS-1025642 to Z. Fei).

\section{LITERATURE CITED}

Alvarez-Venegas, R., Abdallat, A. A., Guo, M., Alfano, J. R., and Avramova, Z. 2007. Epigenetic control of a transcription factor at the cross section of two antagonistic pathways. Epigenetics 2:106-113.

Anders, S., and Huber, W. 2010. Differential expression analysis for sequence count data. Genome Biol. 11:R106.

Benjamini, Y., and Hochberg, Y. 1995. Controlling the false discovery rate: A practical and powerful approach to multiple testing. J. Roy. Stat. Soc. B Met. 57:289-300.

Berardini, T. Z., Mundodi, S., Reiser, L., Huala, E., Garcia-Hernandez, M., Zhang, P., Mueller, L. A., Yoon, J., Doyle, A., Lander, G., Moseyko, N.,
Yoo, D., Xu, I., Zoeckler, B., Montoya, M., Miller, N., Weems, D., and Rhee, S. Y. 2004. Functional annotation of the Arabidopsis genome using controlled vocabularies. Plant Physiol. 135:745-755.

Berr, A., Ménard, R., Heitz, T., and Shen, W. H. 2012. Chromatin modification and remodelling: A regulatory landscape for the control of Arabidopsis defence responses upon pathogen attack. Cell. Microbiol. 14:829-839.

Berr, A., Shafiq, S., and Shen, W. H. 2011. Histone modifications in transcriptional activation during plant development. Biochim. Biophys. Acta 1809:567-576.

Bolger, A. M., Lohse, M., and Usadel, B. 2014. Trimmomatic: A flexible trimmer for Illumina sequence data. Bioinformatics 30:2114-2120.

Boyko, A., and Kovalchuk, I. 2008. Epigenetic control of plant stress response. Environ. Mol. Mutagen. 49:61-72.

Boyko, A., and Kovalchuk, I. 2011. Genetic and epigenetic effects of plantpathogen interactions: An evolutionary perspective. Mol. Plant 4: 1014-1023.

Boyle, A. P., Davis, S., Shulha, H. P., Meltzer, P., Margulies, E. H., Weng, Z., Furey, T. S., and Crawford, G. E. 2008. High-resolution mapping and characterization of open chromatin across the genome. Cell 132: 311-322.

Brabbs, T. R., He, Z., Hogg, K., Kamenski, A., Li, Y., Paszkiewicz, K. H., Moore, K. A., O'Toole, P., Graham, I. A., and Jones, L. 2013. The stochastic silencing phenotype of Arabidopsis morc6 mutants reveals a role in efficient RNA-directed DNA methylation. Plant J. 75:836-846.

Cheng, C., Gao, X., Feng, B., Sheen, J., Shan, L., and He, P. 2013. Plant immune response to pathogens differs with changing temperatures. Nat. Commun. 4:2530.

Czechowski, T., Stitt, M., Altmann, T., Udvardi, M. K., and Scheible, W. R. 2005. Genome-wide identification and testing of superior reference genes for transcript normalization in Arabidopsis. Plant Physiol. 139: 5-17.

De-La-Peña, C., Rangel-Cano, A., and Alvarez-Venegas, R. 2012. Regulation of disease-responsive genes mediated by epigenetic factors: Interaction of Arabidopsis-Pseudomonas. Mol. Plant Pathol. 13:388-398.

Dhawan, R., Luo, H., Foerster, A. M., Abuqamar, S., Du, H. N., Briggs, S. D., Mittelsten Scheid, O., and Mengiste, T. 2009. HISTONE MONOUBIQUITINATION1 interacts with a subunit of the mediator complex and regulates defense against necrotrophic fungal pathogens in Arabidopsis. Plant Cell 21:1000-1019.

Dowen, R. H., Pelizzola, M., Schmitz, R. J., Lister, R., Dowen, J. M., Nery, J. R., Dixon, J. E., and Ecker, J. R. 2012. Widespread dynamic DNA methylation in response to biotic stress. Proc. Natl. Acad. Sci. U.S.A. 109:E2183-E2191.

Feschotte, C. 2008. Transposable elements and the evolution of regulatory networks. Nat. Rev. Genet. 9:397-405.

Furey, T. S. 2012. ChIP-seq and beyond: New and improved methodologies to detect and characterize protein-DNA interactions. Nat. Rev. Genet. 13: 840-852.

Girard, L., and Freeling, M. 1999. Regulatory changes as a consequence of transposon insertion. Dev. Genet. 25:291-296.

Grandbastien, M. A., Audeon, C., Bonnivard, E., Casacuberta, J. M., Chalhoub, B., Costa, A. P., Le, Q. H., Melayah, D., Petit, M., Poncet, C., Tam, S. M., Van Sluys, M. A., and Mhiri, C. 2005. Stress activation and genomic impact of Tnt1 retrotransposons in Solanaceae. Cytogenet. Genome Res. 110:229-241.

Henfrey, R. D., and Slater, R. J. 1988. Isolation of plant nuclei. Methods Mol. Biol. 4:447-452.

Hesselberth, J. R., Chen, X., Zhang, Z., Sabo, P. J., Sandstrom, R., Reynolds, A. P., Thurman, R. E., Neph, S., Kuehn, M. S., Noble, W. S., Fields, S., and Stamatoyannopoulos, J. A. 2009. Global mapping of protein-DNA interactions in vivo by digital genomic footprinting. Nat. Methods 6:283-289.

Ito, H., Gaubert, H., Bucher, E., Mirouze, M., Vaillant, I., and Paszkowski, J. 2011. An siRNA pathway prevents transgenerational retrotransposition in plants subjected to stress. Nature 472:115-119.

Iyer, L. M., Abhiman, S., and Aravind, L. 2008. MutL homologs in restriction-modification systems and the origin of eukaryotic MORC ATPases. Biol. Direct 3:8.

Jones, J. D., and Dangl, J. L. 2006. The plant immune system. Nature 444: 323-329.

Jordan, I. K., Rogozin, I. B., Glazko, G. V., and Koonin, E. V. 2003. Origin of a substantial fraction of human regulatory sequences from transposable elements. Trends Genet. 19:68-72.

Kang, H. G., Foley, R. C., Oñate-Sánchez, L., Lin, C., and Singh, K. B. 2003. Target genes for OBP3, a Dof transcription factor, include novel basic helix-loop-helix domain proteins inducible by salicylic acid. Plant J. 35:362-372. 
Kang, H. G., Hyong, W. C., von Einem, S., Manosalva, P., Ehlers, K., Liu, P. P., Buxa, S. V., Moreau, M., Mang, H. G., Kachroo, P., Kogel, K. H., and Klessig, D. F. 2012. CRT1 is a nuclear-translocated MORC endonuclease that participates in multiple levels of plant immunity. Nat. Commun. 3:1297.

Kang, H. G., and Klessig, D. F. 2005. Salicylic acid-inducible Arabidopsis CK2-like activity phosphorylates TGA2. Plant Mol. Biol. 57: 541-557.

Kang, H. G., Kuhl, J. C., Kachroo, P., and Klessig, D. F. 2008. CRT1, an Arabidopsis ATPase that interacts with diverse resistance proteins and modulates disease resistance to turnip crinkle virus. Cell Host Microbe 3:48-57.

Kang, H. G., Oh, C. S., Sato, M., Katagiri, F., Glazebrook, J., Takahashi, H., Kachroo, P., Martin, G. B., and Klessig, D. F. 2010. Endosomeassociated CRT1 functions early in resistance gene-mediated defense signaling in Arabidopsis and tobacco. Plant Cell 22:918-936.

Kim, K. C., Lai, Z., Fan, B., and Chen, Z. 2008. Arabidopsis WRKY38 and WRKY62 transcription factors interact with histone deacetylase 19 in basal defense. Plant Cell 20:2357-2371.

Krivega, I., and Dean, A. 2012. Enhancer and promoter interactions-long distance calls. Curr. Opin. Genet. Dev. 22:79-85.

Langen, G., von Einem, S., Koch, A., Imani, J., Pai, S. B., Manohar, M., Ehlers, K., Choi, H. W., Claar, M., Schmidt, R., Mang, H. G., Bordiya, Y., Kang, H. G., Klessig, D. F., and Kogel, K. H. 2014. The compromised recognition of turnip crinkle virus1 subfamily of microrchidia ATPases regulates disease resistance in barley to biotrophic and necrotrophic pathogens. Plant Physiol. 164:866-878.

Langmead, B., Trapnell, C., Pop, M., and Salzberg, S. L. 2009. Ultrafast and memory-efficient alignment of short DNA sequences to the human genome. Genome Biol. 10:R25.

Le, T. N., Schumann, U., Smith, N. A., Tiwari, S., Au, P. C., Zhu, Q. H., Taylor, J. M., Kazan, K., Llewellyn, D. J., Zhang, R., Dennis, E. S., and Wang, M. B. 2014. DNA demethylases target promoter transposable elements to positively regulate stress responsive genes in Arabidopsis. Genome Biol. 15:458.

Lewin, R. 1986. "Computer genome" is full of junk DNA. Science 232: 577-578.

Li, H., Handsaker, B., Wysoker, A., Fennell, T., Ruan, J., Homer, N., Marth, G., Abecasis, G., Durbin, R., and 1000 Genome Project Data Processing Subgroup. 2009. The sequence alignment/map format and SAMtools. Bioinformatics 25:2078-2079.

Li, Y., Tessaro, M. J., Li, X., and Zhang, Y. 2010. Regulation of the expression of plant resistance gene $\mathrm{SNC1}$ by a protein with a conserved BAT2 domain. Plant Physiol. 153:1425-1434.

Liu, Z. W., Shao, C. R., Zhang, C. J., Zhou, J. X., Zhang, S. W., Li, L., Chen, S., Huang, H. W., Cai, T., and He, X. J. 2014. The SET domain proteins SUVH2 and SUVH9 are required for Pol V occupancy at RNA-directed DNA methylation loci. PLoS Genet. 10:e1003948.

Lorković, Z. J., Naumann, U., Matzke, A. J., and Matzke, M. 2012. Involvement of a GHKL ATPase in RNA-directed DNA methylation in Arabidopsis thaliana. Curr. Biol. 22:933-938.

Ma, K. W., Flores, C., and Ma, W. 2011. Chromatin configuration as a battlefield in plant-bacteria interactions. Plant Physiol. 157:535-543.

Makarevitch, I., Waters, A. J., West, P. T., Stitzer, M., Hirsch, C. N., RossIbarra, J., and Springer, N. M. 2015. Transposable elements contribute to activation of maize genes in response to abiotic stress. PLoS Genet. 11: e1004915.

Manzara, T., and Gruissem, W. 1995. Identification of promoter sequences that interact with DNA-binding proteins. Pages 233-260 in: Methods in Plant Molecular Biology. A Laboratory Course Manual. P. Maliga, D. F. Klessig, A. R. Cashmore, W. Gruissem, and J. E.Varner, eds. Cold Spring Harbor Laboratory Press, Cold Spring Harbor, NY, U.S.A.

March-Díaz, R., García-Domínguez, M., Lozano-Juste, J., León, J., Florencio, F. J., and Reyes, J. C. 2008. Histone H2A.Z and homologues of components of the SWR1 complex are required to control immunity in Arabidopsis. Plant J. 53:475-487.

Martienssen, R., Barkan, A., Taylor, W. C., and Freeling, M. 1990. Somatically heritable switches in the DNA modification of $\mathrm{Mu}$ transposable elements monitored with a suppressible mutant in maize. Genes Dev. 4:331-343.

Matzke, M. A., and Mosher, R. A. 2014. RNA-directed DNA methylation: An epigenetic pathway of increasing complexity. Nat. Rev. Genet. 15: 394-408.

Moissiard, G., Bischof, S., Husmann, D., Pastor, W. A., Hale, C. J., Yen, L., Stroud, H., Papikian, A., Vashisht, A. A., Wohlschlegel, J. A., and Jacobsen, S. E. 2014. Transcriptional gene silencing by Arabidopsis microrchidia homologues involves the formation of heteromers. Proc. Natl. Acad. Sci. U.S.A. 111:7474-7479.
Moissiard, G., Cokus, S. J., Cary, J., Feng, S., Billi, A. C., Stroud, H., Husmann, D., Zhan, Y., Lajoie, B. R., McCord, R. P., Hale, C. J., Feng, W., Michaels, S. D., Frand, A. R., Pellegrini, M., Dekker, J., Kim, J. K., and Jacobsen, S. E. 2012. MORC family ATPases required for heterochromatin condensation and gene silencing. Science 336:1448-1451.

Moore, J. W., Loake, G. J., and Spoel, S. H. 2011. Transcription dynamics in plant immunity. Plant Cell 23:2809-2820.

Mosher, R. A., Durrant, W. E., Wang, D., Song, J., and Dong, X. 2006. A comprehensive structure-function analysis of Arabidopsis SNI1 defines essential regions and transcriptional repressor activity. Plant Cell 18: 1750-1765.

Naito, K., Zhang, F., Tsukiyama, T., Saito, H., Hancock, C. N., Richardson, A. O., Okumoto, Y., Tanisaka, T., and Wessler, S. R. 2009. Unexpected consequences of a sudden and massive transposon amplification on rice gene expression. Nature 461:1130-1134.

Palma, K., Thorgrimsen, S., Malinovsky, F. G., Fiil, B. K., Nielsen, H. B., Brodersen, P., Hofius, D., Petersen, M., and Mundy, J. 2010. Autoimmunity in Arabidopsis acd11 is mediated by epigenetic regulation of an immune receptor. PLoS Pathog. 6:e1001137.

Pastor, W. A., Stroud, H., Nee, K., Liu, W., Pezic, D., Manakov, S., Lee, S. A., Moissiard, G., Zamudio, N., Bourc'his, D., Aravin, A. A., Clark, A. T., and Jacobsen, S. E. 2014. MORC1 represses transposable elements in the mouse male germline. Nat. Commun. 5:5795.

Perry, J., and Zhao, Y. 2003. The CW domain, a structural module shared amongst vertebrates, vertebrate-infecting parasites and higher plants. Trends Biochem. Sci. 28:576-580.

Probst, A. V., and Almouzni, G. 2011. Heterochromatin establishment in the context of genome-wide epigenetic reprogramming. Trends Genet. 27:177-185.

Quinlan, A. R., and Hall, I. M. 2010. BEDTools: A flexible suite of utilities for comparing genomic features. Bioinformatics 26:841-842.

Robinson, M. D., McCarthy, D. J., and Smyth, G. K. 2010. edgeR: A Bioconductor package for differential expression analysis of digital gene expression data. Bioinformatics 26:139-140.

Sarris, M., Nikolaou, K., and Talianidis, I. 2014. Context-specific regulation of cancer epigenomes by histone and transcription factor methylation. Oncogene 33:1207-1217.

Schmittgen, T. D., and Livak, K. J. 2008. Analyzing real-time PCR data by the comparative C(T) method. Nat. Protoc. 3:1101-1108.

Staiger, D., Korneli, C., Lummer, M., and Navarro, L. 2013. Emerging role for RNA-based regulation in plant immunity. New Phytol. 197:394-404.

Tai, H. H., Tai, G. C., and Beardmore, T. 2005. Dynamic histone acetylation of late embryonic genes during seed germination. Plant Mol. Biol. 59: 909-925.

Tao, Y., Xie, Z., Chen, W., Glazebrook, J., Chang, H. S., Han, B., Zhu, T., Zou, G., and Katagiri, F. 2003. Quantitative nature of Arabidopsis responses during compatible and incompatible interactions with the bacterial pathogen Pseudomonas syringae. Plant Cell 15:317-330.

Thurman, R. E., Rynes, E., Humbert, R., Vierstra, J., Maurano, M. T., Haugen, E., Sheffield, N. C., Stergachis, A. B., Wang, H., Vernot, B., Garg, K., John, S., Sandstrom, R., Bates, D., Boatman, L., Canfield, T. K., Diegel, M., Dunn, D., Ebersol, A. K., Frum, T., Giste, E., Johnson, A. K., Johnson, E. M., Kutyavin, T., Lajoie, B., Lee, B. K., Lee, K., London, D., Lotakis, D., Neph, S., Neri, F., Nguyen, E. D., Qu, H., Reynolds, A. P., Roach, V., Safi, A., Sanchez, M. E., Sanyal, A., Shafer, A., Simon, J. M., Song, L., Vong, S., Weaver, M., Yan, Y., Zhang, Z., Zhang, Z., Lenhard, B., Tewari, M., Dorschner, M. O., Hansen, R. S., Navas, P. A., Stamatoyannopoulos, G., Iyer, V. R., Lieb, J. D., Sunyaev, S. R., Akey, J. M., Sabo, P. J., Kaul, R., Furey, T. S., Dekker, J., Crawford, G. E., and Stamatoyannopoulos, J. A. 2012. The accessible chromatin landscape of the human genome. Nature 489:75-82.

Tittel-Elmer, M., Bucher, E., Broger, L., Mathieu, O., Paszkowski, J., and Vaillant, I. 2010. Stress-induced activation of heterochromatic transcription. PLoS Genet. 6:e1001175.

Vlot, A. C., Dempsey, D. A., and Klessig, D. F. 2009. Salicylic acid, a multifaceted hormone to combat disease. Annu. Rev. Phytopathol. 47: 177-206.

Wang, C., Gao, F., Wu, J., Dai, J., Wei, C., and Li, Y. 2010. Arabidopsis putative deacetylase AtSRT2 regulates basal defense by suppressing PAD4, EDS5 and SID2 expression. Plant Cell Physiol. 51:1291-1299.

Wang, W., Barnaby, J. Y., Tada, Y., Li, H., Tör, M., Caldelari, D., Lee, D. U., Fu, X. D., and Dong, X. 2011. Timing of plant immune responses by a central circadian regulator. Nature 470:110-114.

Watson, M. L., Zinn, A. R., Inoue, N., Hess, K. D., Cobb, J., Handel, M. A., Halaban, R., Duchene, C. C., Albright, G. M., and Moreadith, R. W. 1998. Identification of morc (microrchidia), a mutation that results in arrest of spermatogenesis at an early meiotic stage in the mouse. Proc. Natl. Acad. Sci. U.S.A. 95:14361-14366. 
Wesley, S. V., Helliwell, C. A., Smith, N. A., Wang, M. B., Rouse, D. T., Liu, Q., Gooding, P. S., Singh, S. P., Abbott, D., Stoutjesdijk, P. A., Robinson, S. P., Gleave, A. P., Green, A. G., and Waterhouse, P. M. 2001. Construct design for efficient, effective and high-throughput gene silencing in plants. Plant J. 27:581-590.

Xu, K., Wu, Z. J., Groner, A. C., He, H. H., Cai, C., Lis, R. T., Wu, X., Stack, E. C., Loda, M., Liu, T., Xu, H., Cato, L., Thornton, J. E., Gregory, R. I., Morrissey, C., Vessella, R. L., Montironi, R., Magi-Galluzzi, C., Kantoff, P. W., Balk, S. P., Liu, X. S., and Brown, M. 2012. EZH2 oncogenic activity in castration-resistant prostate cancer cells is Polycomb-independent. Science 338:1465-1469.

Yang, J., and Corces, V. G. 2012. Insulators, long-range interactions, and genome function. Curr. Opin. Genet. Dev. 22:86-92.

Yasuda, K., Ito, M., Sugita, T., Tsukiyama, T., Saito, H., Naito, K., Teraishi, M., Tanisaka, T., and Okumoto, Y. 2013. Utilization of transposable element mPing as a novel genetic tool for modification of the stress response in rice. Mol. Breed. 32:505-516.
Yu, A., Lepère, G., Jay, F., Wang, J., Bapaume, L., Wang, Y., Abraham, A. L., Penterman, J., Fischer, R. L., Voinnet, O., and Navarro, L. 2013. Dynamics and biological relevance of DNA demethylation in Arabidopsis antibacterial defense. Proc. Natl. Acad. Sci. U.S.A. 110:2389-2394.

Zang, C., Schones, D. E., Zeng, C., Cui, K., Zhao, K., and Peng, W. 2009. A clustering approach for identification of enriched domains from histone modification ChIP-Seq data. Bioinformatics 25:1952-1958.

Zaratiegui, M., Irvine, D. V., and Martienssen, R. A. 2007. Noncoding RNAs and gene silencing. Cell 128:763-776.

Zemach, A., Kim, M. Y., Hsieh, P. H., Coleman-Derr, D., Eshed-Williams, L., Thao, K., Harmer, S. L., and Zilberman, D. 2013. The Arabidopsis nucleosome remodeler DDM1 allows DNA methyltransferases to access H1-containing heterochromatin. Cell 153:193-205.

Zhang, W., Zhang, T., Wu, Y., and Jiang, J. 2012. Genome-wide identification of regulatory DNA elements and protein-binding footprints using signatures of open chromatin in Arabidopsis. Plant Cell 24: 2719-2731 